\title{
Psychology of
}

Music

\section{Standardization of a Film Music Stimulus Set (FMSS) to the Spanish Population: Cultural and Gender Differences in the Perception of Emotions Prompted by Music Excerpts}

\begin{tabular}{|r|l|}
\hline Journal: & Psychology of Music \\
\hline Manuscript ID & Draft \\
\hline Keyword: & Music, Discrete emotions, Dimensional approach, Gender, Culture \\
\hline & $\begin{array}{l}\text { The literature review reveals different conceptual and methodological } \\
\text { challenges in the field of music and emotion, such as the lack of } \\
\text { agreement in terms of standardized datasets, or the need of replication } \\
\text { of prior findings. Our study aimed at validating to Spanish population a } \\
\text { set of film music stimulus previously standardized in Finnish samples. } \\
\text { Additionally, we explored the role of gender and culture in the perception } \\
\text { of emotions through music using 102 excerpts selected from the Eerola } \\
\text { \& Vuoskoski's dataset. A total of 129 voluntary undergraduate students } \\
\text { (71,32\% females) from different degrees participated voluntarily in this } \\
\text { study, where they were instructed to rate both discrete emotions } \\
\text { (Happiness, Sadness, Tenderness, Fear, Anger), as well as affective } \\
\text { dimensions (Valence, Energy Arousal, Tension Arousal) using a 9-point } \\
\text { scale after presentation of each excerpt. Strong similarities between } \\
\text { Finnish and Spanish ratings were found, with only minor discrepancies } \\
\text { across samples in the evaluation of basic emotions. Taken together, our } \\
\text { findings suggest that normative ratings for Spanish and Finnish } \\
\text { participants across gender are released, so the current database is } \\
\text { suitable for research on music and emotions. Additional theoretical and } \\
\text { practical implications of this validation are discussed. }\end{array}$ \\
\hline
\end{tabular}

\section{SCHOLARONE" Manuscripts}




\begin{abstract}
The literature review reveals different conceptual and methodological challenges in the field of music and emotion, such as the lack of agreement in terms of standardized datasets, or the need of replication of prior findings. Our study aimed at validating to Spanish population a set of film music stimulus previously standardized in Finnish samples. Additionally, we explored the role of gender and culture in the perception of emotions through music using 102 excerpts selected from the Eerola \& Vuoskoski's dataset. A total of 129 voluntary undergraduate students $(71,32 \%$ females $)$ from different degrees participated voluntarily in this study, where they were instructed to rate both discrete emotions (Happiness, Sadness, Tenderness, Fear, Anger), as well as affective dimensions (Valence, Energy Arousal, Tension Arousal) using a 9-point scale after presentation of each excerpt. Strong similarities between Finnish and Spanish ratings were found, with only minor discrepancies across samples in the evaluation of basic emotions. Taken together, our findings suggest that normative ratings for Spanish and Finnish participants across gender are released, so the current database is suitable for research on music and emotions. Additional theoretical and practical implications of this validation are discussed.
\end{abstract}

Keywords: music, discrete emotions, dimensional approach, gender, culture 


\section{Introduction}

The study of human emotions has generated an interesting scientific debate for decades (Lepping, Atchley \& Savage, 2016). In laboratory contexts, different affective stimuli have been used as reliable tools to induce emotions, such as pictures (IAPS: Lang, Bradley \& Cuthbert, 1999), sounds (IADS: Bradley \& Lang, 2007), words (ANEW: Bradley \& Lang, 2017), or facial expressions (POFA: Ekman \& Friesen, 1976). All these stimuli are generally part of standardized datasets that provide normative values in different affective dimensions (e.g., hedonic valence and arousal), and have been used in numerous experimental procedures, including passive exposure to affective stimuli (Bradley, Codispoti, Cuthbert \& Lang, 2001; Ciuffini, Stratta \& Marrelli, 2018), emotional anticipation (Sege, Bradley \& Lang, 2014), or emotion regulation (Bernat, Cadwallader, Seo, Vizueta \& Patrick, 2011; Conzelmann, Mcgregor \& Pauli, 2015; Fuentes-Sánchez, Jaén, Escrig, Lucas \& Pastor, 2019). The use of these stimuli has been essential in the study of brain function (Bermpohl et al., 2006; Ozawa, Kanayama \& Hiraki, 2019), peripheral psychophysiological correlates (Gomez, Gunten \& Danuser, 2016), as well as subjective evaluations (Bradley et al., 2001) that are part of emotional reactions. Indeed, the scientific study of human emotion has advanced, to a large extent, based on empirical findings obtained using standardized stimuli, which has allowed for replicability of results across different laboratory contexts.

Regarding the use of music as emotional stimuli, however, the literature reveals important methodological and conceptual caveats. Firstly, a variety of musical genres have been used due to the lack of consensus among the scientific community on the best type of stimuli to induce emotions. To this extent, past research has mostly focused on instrumental classical music (Juslin \& Laukka, 2003; Kreutz, Ott, Teichmann, Osawa \& Vaitl, 2008), besides other types of music such as pop (Song, Dixon, Pearce \& Halpern, 2016), mixed genres (Imbir \& Golab, 2017), or film soundtracks (Eerola \& Vuoskoski, 2011). Secondly, 
different theoretical conceptualizations of emotions (i.e., discrete $v s$. dimensional models) have been proposed over the last two decades (Eerola \& Vuoskoski, 2011; Song et al., 2016). Although both models are complementary and have gained support in the field of music and emotion research (Gomez \& Danuser, 2004; Imbir \& Golab, 2017), recent studies suggest that the dimensional model is more reliable in comparison to the discrete or categorical model, specifically when ambiguous examples of an emotion category are rated (Eerola \& Vuoskoski, 2011). Thirdly, only a few standardized musical stimuli databases are available despite their advantages to enhance reproducibility of emotion research (Eerola \& Vuoskoski, 2011; Imbir \& Golab, 2017; Lepping et al., 2016; Song et al., 2016; Vieillard, Peretz, Gosselin, Khalfa, Gagnon \& Bouchard, 2008). This lack of extensive research using normative stimuli can also lead to further challenges when comparing findings across laboratories (Dienes, 2008; Frieler, Müllensiefen, Fischinger, Schlemmer, Jakubowski \& Lothwesen, 2013).

In addition to the above-mentioned methodological and theoretical caveats, the role of gender differences has been scarcely explored in the context of music and emotion (Imbir \& Golab, 2017). Whereas prior findings with affective pictures have also shown that women tend to rate unpleasant stimuli as more arousing than men (Bradley, Greenwald \& Hamm, 1993; Greenwald, Cook \& Lang, 1989; Moltó et al., 1999, 2013; Vila et al., 2001), gender does not seem to influence emotional responses to sounds (Fernández-Abascal et al., 2008), words (Redondo et al., 2007) or music (Imbir \& Golab, 2017). To this extent, further research is needed to elucidate the precise involvement of gender effects in music-induced emotional responses.

On the other hand, plausible cultural differences should be considered for a greater understanding of emotional perception of music, as already shown in diverse sensory modalities. Past research has compared subjective evaluations in Anglo-Saxon and Spanish 
populations with affective pictures (Moltó et al., 1999, 2013; Vila et al., 2001), sounds (Fernández-Abascal et al., 2008), and words (Redondo, Fraga, Padrón \& Comesaña, 2007; Redondo, Fraga, Padrón \& Piñeiro, 2008). In general, these studies have found differences regarding arousal but not valence ratings (Fernández-Abascal et al., 2008; Moltó et al., 1999, 2013; Vila et al., 2001). In particular, more extreme arousal ratings in Spanish compared to Anglo-Saxon population have been reported. Similarly, a few cross-cultural studies using music stimuli reveal relevant differences in the recognition of some basic emotions. For example, Argstatter (2015) showed that Western European listeners are characterized by better recognition of basic emotions (anger, disgust, fear, happiness, sadness, and surprise) than Asian participants. However, some of that discrete emotions such as anger, disgust, fear, and surprise were easily confused in both groups. Furthermore, other studies focused on specific basic emotions such as anger (Susino \& Schubert, 2017; Kwuon, 2009), showed that music depicting anger mood was poorly decoded by Indian, Japanese, and Swedish listeners, possibly due to stereotypes associated with their specific cultures. Therefore, the literature review suggests that cross-cultural communication of some basic emotions might not be reliable across and within some cultures, pointing out to the need of further research focused on cultural differences using also dimensional models instead of discrete model of emotions only.

The present study selected a representative sample of Spanish population to validate a standardized database of musical excerpts - previously validated in Finland by Eerola \& Vuoskoski (2011)-, aiming to extend prior findings by exploring both cultural and gender differences. Among currently available music stimuli datasets, we selected the one proposed by Eerola \& Vuoskoski (2011) since it was specifically designed to induce powerful emotion responses while controlling both familiarity and preference. Our study aimed at providing the Spanish scientific community, or by cultural affinity other countries in southern Europe, with 
a set of musical stimuli with normative values in different affective dimensions (valence, energy, tension), as well as discrete emotions (happiness, sadness, anger, tenderness, fear), separately for men and women. This additional information might facilitate the selection of musical excerpts for future basic and clinical studies (e.g., those seeking to explore induction and/or regulation of emotions through music). Furthermore, we aimed at replicating the results obtained in the original study (Eerola \& Vuoskoski, 2011) regarding the suitability of the dimensional and categorical models. According to prior literature focused on standardized affective stimuli (Fernández-Abascal et al., 2008; Moltó et al., 1999, 2013; Vila et al., 2001), we expected to find a strong agreement between Spanish and Finnish populations, as well as between women and men, for the evaluation of basic emotions and hedonic valence ratings, along with a few differences in terms of arousal ratings.

\section{Methods}

\section{Participants}

A total of 136 participants (69.85\% females) between 18 and 50 years (Mean age $=$ $22.04, \mathrm{SD}=4.63$ ) enrolled voluntarily in this study. The sample size was based on a power analysis using G*Power (Faul, Erdfelder, Lang, \& Buchner, 2007). We needed a minimum of 115 participants with an $95 \%$ chance to detect a small-to-medium sized effect $\left(\mathrm{f}^{2}=.15\right)$ at an alpha level of .05. The sample was composed by undergraduate students of different knowledge branches (science, humanities, engineering, social sciences and health science) at Universitat Jaume I (Castellón de la Plana, Spain). Seven participants were excluded due to technical problems during data acquisition. As a result, statistical analyses were performed with a total of 129 (71.32\% females) participants. Ethical approval from the Deontological Comision at Universitat Jaume I was obtained, and all participants provided written informed consent forms.

\section{Stimuli and Design}


Although the original dataset contains 110 film music excerpts (available at https://osf.io/p6vkg/), for this research we selected only 102 pieces due to the exclusion of duplicated stimuli. Excerpt duration ranged between 11 and 31 seconds $(M=17.63, S D=$ 3.73), and music did not contain lyrics, dialogue or sound effects (Eerola \& Vuoskoski, 2011). These excerpts were distributed into two sets of five blocks with ten excerpts each one, except two blocks of 11 excerpts, with no more than two consecutive excerpts that convey similar emotions. Both sets did not differ a priori in the normative values obtained in Eerola \& Vuoskoski study (2011) in any affective scale (all $t s<1)$ (see Table 1).

\section{[TABLE 1 NEAR HERE]}

Participants were assigned randomly into two groups. Group $1(n=64)$ started with 51 excerpts rated using a scale 1-9 for each discrete emotion (Set 1), followed by 51 excerpts rated using a bipolar scale of 1-9 for each three axes of dimensional model (Set 2). Group 2 $(n=65)$ started with the excerpts of Set 2 rated with discrete model followed by excerpts of Set 1 rated with dimensional model. Each excerpt was therefore assessed following both the discrete and dimensional models of emotion, but neither excerpt was assessed using both emotion models by the same participant. Additionally, the order of the blocks within each set was randomized individually for each participant.

\section{Apparatus}

Auditory stimuli were presented using E-Prime 2.0 software (Psychology Software Tools, Inc. Scharpsburg, PA) on a standard 17-inch computer monitor, and responses were collected using a small keyboard. Musical excerpts were played through Sennheiser HD-205 headphones, which provided professional sound quality and reduced external noise. Volume was kept constant across participants, being determined by a pilot experiment.

\section{Procedure}


Each subject participated individually in one laboratory session, which lasted approximately $1 \mathrm{~h}$ and $30 \mathrm{~min}$. First, participants read an overview of the task and completed a written consent form. Afterwards, they completed a survey to collect individual variables including age, gender, educational level, history of musical training or hearing problems. Before the experiment, participants were trained to differentiate between perceived and induced emotions, and how to rate the emotions in each music excerpt using different scales. To ensure that they had understood the procedure, participants were asked to rate 2 music examples (selected from Vieillard et al., 2008). The experimental task was divided into two parts, separated by a short break (2-5 min) with the aim of keeping their attention throughout the whole experiment. During the first part, participants had to rate each musical excerpt using a 9-point scale for each discrete emotion (happiness, sadness, tenderness, fear, anger). In the second part, they were instructed to rate each musical excerpt using a 9-point scale for each affective dimension (valence, energy arousal, tension arousal). In both parts of the experiment, participants were also asked to rate their "preference" using a 9-point scale (i.e., how much they liked each stimulus), as well as their "familiarity" using a 3-point scale $(0=$ unfamiliar, $1=$ somewhat familiar, $2=$ very familiar $)^{1}$. Participants had to complete their assessments for all the above scales (discrete emotions or affective dimensions, plus preference and familiarity) for each excerpt before they were allowed to continue. After rating all the musical excerpts, participants were debriefed about the purpose of the experiment.

\section{Data analysis}

Descriptive statistics (Mean and Standard Deviation) were calculated for each musical excerpt (for the overall sample, as well as separately for women and men). Independent samples $t$-test analyses were performed to examine gender differences. A correlational analysis was conducted to assess how both conceptual frameworks (dimensional vs. 
categorical or discrete) could be used to describe perceived emotions and to clarify what type of dimensional model would be the most appropriate for such studies. Moreover, a partial correlation analysis was conducted in order to examine the collinearity between different emotion concepts, controlling for the contribution of other discrete categories or dimensions. In this vein, we conducted partial correlation analyses between discrete emotions and affective dimensions.

In order to explore the correspondence between the discrete and dimensional models, multiple regression analyses were performed with the aim of predicting the dimensional ratings from the discrete ratings, and vice-versa. More specifically, we conducted a regression analysis with the categorical model (happiness, sadness, tenderness, anger and fear) as a predictor, and each dimensional variable (valence, energy and tension arousal) as independent measures. Thereupon, we performed an additional regression analysis with the dimensional model as a predictor and each discrete emotion as independent measures.

Finally, $t$ test analysis was carried out to investigate the differences between Finnish and Spanish populations in the different scales evaluated in the study.

\section{Results}

\section{Normative ratings in Spanish population}

Mean $(M)$ and Standard Deviation $(S D)$ for each musical excerpt and for the overall sample are summarized in Table 1 (see Appendix), for women (Table 2, Appendix) and for men (Table 3, Appendix). These data constitute the normative values of Spanish standardization.

In Table 2, Mean $(M)$, Standard Deviation $(S D)$ and Confidence Interval $(C I)$ for each scale are presented separately for women and men, besides the result of the $t$ test comparison between both genders. Women assigned higher scores to anger, fear, energy arousal and 
tension arousal, all $p s<.05$ (see Table 2), whereas men assigned higher scores to happiness, tenderness, sadness and valence all $p s<.05$.

\section{[TABLE 2 NEAR HERE]}

\section{Replication of previous findings}

Pairwise correlations between emotion concepts

Relationships between the dimensions investigated were analysed using correlations (Pearson's $r$ ). All the correlations have been summarized in Table 3 but only those significant are discussed here. Regarding the discrete emotions, results showed strong positive correlations between fear and anger, $r(6)=.86, p<.001$, replicating previous results from Eerola \& Vuoskoski study who obtain a similar result. Concerning tenderness, we observed a significant positive correlation both with sadness, $r(6)=.34, p<.001$, and happiness, $r(6)=$ $.57, p<.001$. Additionally, strong negative correlations were found between tenderness and both fear and anger, $r(6)=-.83, p<.001$ for both, suggesting that those excerpts that reflected better these discrete negative emotions were also perceived as less tenderness.

With respect to affective dimensions, a positive relationship was observed between valence and happiness, $r(6)=.87, p<.001$, but not between valence and sadness, $r(6)=-.06$, $p>.05$. Additionally, we found strong negative correlations between valence and fear, $r(6)=$ $-.95, p<.001$, as well as between valence and anger, $r(6)=-.82, p<.001$, showing that those excerpts rated as more pleasant were also perceived as less negative emotions such as fear or anger-

In addition, a strong positive correlation was observed between tension arousal and energy arousal, $r(6)=.89, p<.001$, suggesting a possible overlap between both dimensions. Furthermore, negative correlations between valence and each arousal dimension were found, being more significant for tension arousal, $r(6)=-.68, p<.001$, than for energy arousal, $r(6)$ $=-.31, p<.01$. 


\section{[TABLE 3 NEAR HERE]}

\section{Partial correlations between emotion concepts}

As we shown in Table 4, results showed negative and highly significant correlations between happiness and sadness, $r(3)=-.91, p<.001$, as well as between happiness and fear, $r(3)=-.73, p<.001$, showing that the happiest excerpts were also rated low either in sadness and fear. Additionally, partial correlations showed that association between fear and anger were not significant, $r(3)=.05, p>.05$, in contrast with the pairwise correlations in which there was a strong association between both concepts, $r(3)=.86, p<.001$.

\section{[TABLE 4 NEAR HERE]}

Regarding the three-dimensional model, partial correlations showed a high overlap between energy and tension arousal, $r(1)=.98, p<.001$, when partialling out the contribution of the valence, which suggests the possibility to collapse into a single arousal dimension. Lastly, partial correlation between valence and both energy and tension arousal dimensions was even stronger when the energy or tension ratings were partialled out, $r(1)=.92, p<.01$ for energy arousal; $r(1)=-.95, p<.001$ for tension arousal.

\section{Correspondence between discrete and dimensional models of emotion}

Regressions suggested that the categorical model would explain a high percentage of variance of the dimensional model, and vice-versa (See $R^{2}$ in Table 5). Particularly, the former (happiness, sadness, tenderness, anger and fear) was highly significant to predict the latter (all $p \mathrm{~s}<.0001$ ). Regarding valence ratings, the best predictor within the categorical model was fear, $F(1,96)=42.59, p<.0001, \eta^{2}{ }_{p}=.31$, followed by tenderness, $F(1,96)=$ $13.48, p<.001, \eta_{p}^{2}=.12$. On the other hand, the best predictor for energy arousal was tenderness, $F(1,96)=85.78, p<.0001, \eta_{p}^{2}=.47$, followed by anger, $F(1,96)=78.02, p<$ $.0001, \eta_{p}^{2}=.45$, and happiness, $F(1,96)=36.10, p<.0001, \eta^{2}=.27$. Finally, tenderness was 
also the best predictor for tension arousal, $F(1,96)=99.33, p<.0001, \eta^{2}=.51$, followed by anger, $F(1,96)=53.59, p<.0001, \eta_{p}^{2}=.36$.

Regarding the dimensional model as a predictor, findings showed that this model was highly significant for all categorical ratings $(p s<.0001)$. Firstly, with regards to happiness, the best predictor was valence ratings, $F(1,98)=30.88, p<.0001, \eta_{p}^{2}=.24$, followed by energy arousal, $F(1,98)=27.31, p<.0001, \eta_{p}^{2}=.22$, and tension arousal, $F(1,98)=10.91, p$ $=.001, \eta_{p}^{2}=.10$. Valence also was the best predictor for anger, $F(1,98)=6.38, p=.013, \eta_{p}^{2}$ $=.06$, fear, $F(1,98)=30.75, p<.0001, \eta_{p}^{2}=.24$, and sadness, $F(1,98)=7.00, p<.001, \eta_{p}^{2}=$ .07 . In contrast, the best predictor for tenderness was the tension arousal, $F(1,98)=8.18, p<$ $.001, \eta_{p}^{2}=.08$

\section{[TABLE 5 NEAR HERE]}

\section{Comparison between Spanish and Finnish evaluations}

Descriptive statistics and correlations between populations

Table 6 summarizes descriptive statistics for the different categorical and dimensional scales, as well as $t$ test between both populations. Specifically, Spanish population scored the excerpts higher in all emotions in comparison with Finnish. On the other hand, regarding dimensional model, results showed that both populations differed in the evaluation of energy and tension arousal, but not in valence. Specifically, Finnish population rated the excerpts as more energetic and tense in comparison with Spanish population.

\section{[TABLE 6 NEAR HERE]}

Additionally, the correlations between the mean ratings between the two samples was yield highly significant across the different dimensional ratings $(r(4)=.96, p<.001$ for valence; $r(4)=.93, p<.001$ for energy arousal; and $r(4)=.94, p<.001$ for tension arousal) and categorical ratings $(r(8)=.94, p<.001$ for happiness; $r(8)=.94, p<.001$ for anger; $r(8)$ $=.96, p<.001$ for fear; $r(8)=.93, p<.001$ for tenderness; $r(8)=.91, p<.001$ for sadness). 


\section{Overlap of the two models of emotion in Spanish and Finnish population}

\section{[FIGURE 1 NEAR HERE]}

Figure 1a shows the relationship between valence and energy arousal, valence and tension arousal (Figure 1b), and energy and tension arousal (Figure 1c) for Spanish and Finnish population. As shown in Figure 1a, unpleasant stimuli were necessarily rated as arousing in order to be perceived as negative. However, this result was not replicated in the positive pole, where some excerpts were rated either less or high arousing. Particularly, high arousing excerpts convey happiness whereas the low arousing clips convey tenderness, but both type of clips have similar valence score (see Figure 1a). Furthermore, both results are highly similar in Spanish and Finnish population. There are slight differences between both populations in the excerpts rated as more pleasant, which were rated by Finnish as more energetic compared to Spanish participants. On the other hand, the relation between valence and tension (see Figure 1b) was negative for both populations, showing that those excerpts rated as positive also was evaluated as less tense. Particularly interesting is the clear distinction of the basic emotions in the graph. Clearly, the least tense were tenderness excerpts (but not happiness, which were evaluated as medium in tension arousal), whereas the most tense were anger and fearful clips. Finally, regarding the relation between tension and energy arousal (see Figure 1c), a strong relationship was found in both samples, being more pronounced for Spanish population. For Finnish population, the data were more dispersed, particularly in those excerpts that convey happiness and sadness, which were rated as more energetic and less energetic, respectively, as compared to Spanish population.

\section{Discussion}

Eerola and Vuoskoski (2011) presented a rich, systematically structured dataset designed to investigate music-associated emotions. Their study not only introduced a valuable researcher-oriented set of film music stimulus but also compared the categorical and 
dimensional approaches to the study of emotion in music perception. Employing the same stimulus material, the present study supports and extends the notion that this soundtrackbased music can be effectively used as a comprehensive and powerful research tool in the field of human emotion. Our findings reveal a distribution of excerpts across the bidimensional affective space that highly resemble other emotional stimuli modalities, such as words, pictures or sounds (Carretié, Tapia, López-Martín \& Albert, 2019; FernándezAbascal et al., 2008; Kurdi, Lozano \& Banaji, 2017; Marchewka, Zurawski, Jwsnoróg \& Grabowska, 2014; Moltó et al., 1999, 2013; Redondo et al., 2007). Furthermore, this validation provides the scientific community with new insights about music-induced emotions in Spanish population, likewise other affective stimuli previously adapted to this cultural context and broadly used in the last decades in experimental research (Carretié et al., 2019; Fernández-Abascal et al., 2008; Moltó et al., 1999, 2013; Redondo et al., 2007; Vila et al., 2001). In addition, this music database has the advantage over other sets of affective stimuli that allow to select the excerpts not only in terms of valence and arousal ratings (as in the case of pictures, sounds or words), but also considering their normative values in different basic emotions (such as happiness, fear or anger, among others).

\section{Gender differences in emotion perception through music}

Standardization studies of music databases have generally provided normative values for the overall sample without exploring gender-influenced discrepancies in subjective ratings (Bigand, Vieillard, Madurell, Marozeau \& Dacquet, 2005; Lepping et al., 2016; Paquette, Peretz \& Belin, 2013). In fact, most of the studies do not even report the percentage of women in their samples (Bigand et al., 2005; Lepping et al., 2016), difficulting the possibility to draw conclusions about plausible differences between women and men.

In this regard, the literature review reveals a mixed picture, depending on the modality of affective stimuli or the theoretical approximation to emotion. Thus, a few studies 
reported gender differences (Bradley, Codispoti, Sabatinelli \& Lang, 2001b; Moltó et al., 1999, 2013; Vila et al., 2001), whereas some other works did not (Grimshaw, BulmanFleming \& Sgo, 2004; Rahman, Wilson \& Abrahams, 2004). In the current study, women rated music excerpts as more fearful, angry, and arousing compared to men. These findings go partially in line with previous works in which women evaluated affective stimuli as more arousing, especially unpleasant pictures, suggesting a possible bias toward the negative pole (Bradley, et al., 2001b; Carretié et al., 2019; Moltó et al., 1999, 2013; Vila et al., 2001). In addition, prior studies using facial expressions showed that women were more prone to recognize emotions such as anger, disgust, fear and sadness (Duesenberg et al., 2016; Hall \& Matsumoto, 2004). This set of results have clinical implications since gender-influenced differences in emotion processing have been suggested to play an important role in serious disorders such as anxiety and depression (Nolen-Hoeksema \& Aldao, 2011). Given the increase in the therapeutic use of music in pathologies characterized by underlying emotional problems such as chronic pain, fibromyalgia, dementia, or autism spectrum disorders (Thompson, 2015), the present standardization to Spanish population (which provides normative ratings for the global sample but also for women and men), might be a remarkable contribution to clinical researchers.

\section{Replication of main findings in Eerola \& Vuoskoski (2011)}

Similar results to the original study by Eerola \& Vuoskoski (2011) were overall found, among which stands out the strong correlation between anger and fear ratings, suggesting certain difficulties distinguishing between both negative emotions in the music domain. This result has been previously reported (Eerola \& Vuoskoski., 2011; Kallinen \& Ravaja, 2006; Vieillard et al., 2008), unlike what happens with other basic emotions such as happiness or sadness, certainly easier to be perceived and distinguished from each other through musical excerpts (Laukka, Eerola, Thingujam, Yamasaki \& Beller, 2013). In 
addition, it has also been suggested that the lack of capacity of music to convey and induce particular emotions such as fear might explain this difficulty to distinguish among specific emotions. To this extent, Vieillard et al (2008) stated that flight or fight overt responses that are necessarily present in fear might not be truly induced by musical stimulation. Conversely, fMRI studies suggest that fear music is able to activate the amygdala region (Gosselin, Peretz, Johnen \& Adolphs, 2007), likewise other affective modalities (Aalto et al., 2002; Sander \& Scheich, 200). In fact, previous works (Gosselin et al., 2005; Gosselin, Peretz, Johnsen \& Adolphs, 2007) have demonstrated that the perception of fear through music can disappear after amygdala damage, suggesting the role of the limbic system in the perception of basic musical emotions.

Regarding the positive pole, a strong relation between tenderness and happiness was found in the current study, in line with previous works reporting similar associations between positive emotions (Juslin, 2001). Interestingly, this result was found in Spanish but not in Finnish population (Eerola \& Vuoskoski, 2011). In addition, it seems remarkable the relationship between the subjective ratings in valence, happiness and sadness. Thus, whereas happy music was evaluated as positive (or pleasant), according to previous results (Eerola $\&$ Vuoskoski, 2001), sad music was not rated as negative (or unpleasant) music unlike other basic emotions such as fear and anger. This result might be related to the "paradox of enjoying sad music" (Eerola, Vuoskoski, Peltola, Putkinen \& Schäfer, 2018), which has been explored at different levels of analyses, such as neurochemical (Huron, 2011), brain imaging (Mitterschiffthaler, Fu, Dalton, Andrew \& Williams, 2007; Trost, Ethofer, Zentner \& Vuilleumier, 2008), psychosocial (Saarikallio \& Erkkilä, 2007; Van Goethem \& Sloboda, 2011) or cultural viewpoints (Mesquita \& Walker, 2003), in order to give an explanation for the pleasure experienced when listening to of sad music. In sum, the literature review reveals the existence of crucial gaps in the understanding of this interesting paradox, besides the need 
of new experimental studies to complete the puzzle (Eerola et al., 2018). In this vein, we would like to highlight the significance of reproducibility in scientific psychology, as well as the broader field of cognitive and affective neuroscience (Frieler, Müllensiefen, Fischinger, Schlemmer, Jakubowski \& Lothwesen, 2013). This requirement is even more important within the scope of empirical studies exploring emotion perception through music due to the relative novelty of this research area, in contrast to other well-tested paradigms such as the passive viewing of affective pictures, widely explored in the field of emotion induction and regulation.

\section{Categorical and Dimensional Models of Emotion in Music Perception}

Over the last two decades, both discrete and dimensional models have been used in the study of music and emotion (Song et al., 2016). According to the discrete (categorical) approach, all emotions can be derived from a limited number of basic universal and innate emotions, such as fear, happiness, anger, disgust and sadness (Ekman, 1992). The dimensional model, however, considers that all emotions can be understood as variants of hedonic valence and affective intensity (Bradley \& Lang, 2007; Wundt, 1896). The latter perspective considers that all emotions arise from two independent neurophysiological systems: hedonic valence (unpleasant-pleasant) and arousal (activation-deactivation) (Eerola \& Vuoskoski, 2011).

Our results showed a strong correspondence between models, supporting the previously proposed notion that both theoretical approaches are complementary (Eerola \& Vuoskoski, 2011; Gomez \& Danuser, 2004). The combination of both models could allow distinguishing specific emotions that might be ignored when considering only the dimensional model, such as anger and fear (which are evaluated as equally unpleasant and arousing stimuli). This is also applicable for neutral stimuli (generally perceived as neutral in valence and low arousing), not fitting as real neutral stimuli from the categorical perspective. 
Instead, results showed that those stimuli convey sadness. Additionally, the combination of both emotion approaches could overcome the limitations that each model can have separately, like for example the difficulty of focusing on basic emotions in cross-cultural studies due to linguistic features or peculiarities for understanding specific emotions in each culture, in function of their particular social rules. Therefore, future studies using music as emotional stimuli should consider integrating both theoretical approximations since it may become more appropriate to describe the richness of music-induced emotion (Song et al., 2016).

Regarding the dimensional model, there is currently an open debate about the number of dimensions that need to be included. Different statistical and theoretical proposals have suggested the use of either one (Russell, 1980) or two - energy and tension-arousal dimensions (Schimmack \& Grob, 2000; Schimmack \& Reisenzein, 2002). Our findings showed a strong relationship between both arousal dimensions, suggesting that they could be collapsed into one unique dimension, as suggested by the two-dimensional model (Rusell, 1980) and previously reported by other researchers (Eerola \& Vuoskoski, 2011). However, the question now is which arousal dimension should be considered in future research, or whether it is necessary to combine both in one single dimension. To this extent, both arousal dimensions are highly similar but their relationship with valence seems quite different. Whereas the bidimensional affective space with valence and energy arousal replicated the boomerang effect previously found with other type of stimulus (Carretié et al., 2019; Fernández-Abascal et al, 2008; Moltó et al., 1999, 2013; Redondo et al., 2007; Vila et al., 2001), the distribution with valence and tension arousal showed a negative, linear relationship. Together with the original study (Eerola \& Vuoskoski, 2011), our data demonstrate that the dimension of energy arousal might be more similar to that explored in past studies (Imbir \& Golab, 2017; Lepping et al., 2016). This notwithstanding, it is an open 
question whether it could be better to combine both arousal dimensions or to simply use energy arousal, and this will need to be clarified in future studies.

\section{Cultural differences in Emotion Perception through Music}

According to our hypothesis, Spanish and Finnish participants were quite similar in the evaluation of the music excerpts as reflected in the strong correlations between different affective dimensions and categories. These results demonstrate the utility of this database to convey similar emotions in different countries. Furthermore, our findings show the universal capacity to identify emotions through music, in a similar way to the recognition of emotional prosody (Fritz et al., 2009).

Nevertheless, we appreciated some peculiarities among both populations. Findings showed that Finnish participants rated the excerpts as more energetic and tense, in comparison with Spanish, but these differences are nuanced when the categorical model was taken into account. Particularly, happy excerpts were evaluated as more energetic by Finnish population, but other excerpts that convey other emotions (such as sad) were rated as less energetic by Finnish in comparison with Spanish population. These results do not confirm previous findings focussed in the dimensional model, in which Spanish people rated the stimuli as more arousing compared to American population (Moltó et al., 1999, 2013; Redondo et al., 2007; Vila et al., 2001). However, it is worth noting that differences between Spanish and Anglo-saxons are not found using emotional sounds (Fernandez-Abascal et al., 2008), suggesting that the sensory modality of the stimuli -or cultural conventions- could influence the emotional perception across cultures. Regarding the categorical model, Spanish population rated the excerpts as more emotional in all basic emotions. Thus, the categories of emotion used in this experiment could influence the above results. Thus, while broad dimensions such as valence and arousal may be applicable across cultures, specific emotional categories may not be properly translated among cultures (Thompson \& Balkwill, 2010). For 
instance, the word of anger could have different interpretations by members of different cultures or countries, depending on the specific norms for expressing and interpreting emotions.

\section{Limitations and future directions}

The current study is not exempt from methodological limitations that should be taken into account when interpreting our findings. Firstly, the number of male and female was unbalanced compared to prior studies investigating gender differences using stimuli from another sensory modalities (Fernández-Abascal et al., 2008; Moltó et al., 1999, 2013; Redondo et al., 2007). Secondly, we have not explored individual variables that could affect the evaluations, such as music preference or expertise (Bigand et al., 2005). The third limitation concerns the range scales used in the experiment, which were of 9-points and anchorages were represented to the participants based on adjectives likewise in the original study. The use of language in affective ratings might involve a problem because the language is not free of cultural differences (Moltó et al., 1999). In fact, some cultures have highly expressive terms for certain emotional concepts, meanwhile others have a lack of terms for expressing specific emotional concepts, which may affect emotion recognition across cultures (Thompson \& Balkwill, 2010). Therefore, future studies should use other rating procedures free from effects of language such as the Self-Assessment Manikin (SAM; Lang, 1980), which would allow to compare directly with other affective stimuli (Fernández-Abascal et al., 2008; Vila et al., 2001).

Additional limitations due to certain features of this music database should be considered. On the one hand, this database contains fewer stimuli compared with other emotional datasets (such as IAPS, IADS or EmoMadrid), which could complicate the selection of certain exemplars for future studies according to specific criteria of each experimental design. Furthermore, music excerpts included in this database vary in duration, 
which could clearly influence the results in psychophysiological experiments where this feature might be a relevant methodological factor to take into consideration. Consequently, future studies might validate this database using the same duration for all the clips, adding also further musical excerpts following the same procedure reported in Eerola \& Vuoskoski (2011) and accurately replicated here. 


\section{References}

Aalto, S., Näätänen, P., Wallius, E., Metsähonkala, L., Stenman, H., Niem, P.M., \& Karlsson, H. (2002). Neuroanatomical substrata of amusement and sadness: a PET activation study using film stimuli. Neuroreport, 13(1), 67-73. doi:10.1097/00001756-200201210-00018

Argstatter, H. (2015). Perception of basic emotions in music: Culture-specific or multicultural? Psychology of Music, 44(4), 1-17. doi:10.1177/0305735615589214

Baumgartner, T., Esslen, M., \& Jäncke, L. (2006). From emotion perception to emotion experience: Emotions evoked by pictures and classical music. International Journal of Psychophysiology, 60(1), 34-43. doi:10.1016/j.ijpsycho.2005.04.007

Bermpohl, F., Pascual-Leone, A., Amedi, A., Merabet, L.B., Fregni, F, Gaab, N.,... Northoff, G. (2006). Attentional modulation of emotional stimulus processing: an fMRI study using emotional expectancy. Human Brain Mapping, 27(8), 662-677. doi:10.1002/hbm.20209

Bernat, E. M., Cadwallader, M., Seo, D., Vizueta, N., \& Patrick, C. J. (2011). Effects of Instructed Emotion Regulation on Valence, Arousal, and Attentional Measures of Affective Processing. Developmental Neuropsychology, 36(4), 493-518. doi:10.1080/87565641.2010.549881

Bigand, E., Vieillard, S., Madurell, F., Marozeau, J., \& Dacquet, A. (2005). Multidimensional scaling of emotional responses to music: The effect of musical expertise and of the duration of the excerpts. Cognition and Emotion, 19(8), 1113-1139.

doi:10.1080/02699930500204250

Bradley, M. M. (2000). Emotion and Motivation. In J. T. Cacioppo, L. G. Tassinary \& Berntson, G. G. (Eds.): Handbook of Psychophysiology (pp. 602-642). New York, USA: Cambridge University Press.

Bradley, M. M., \& Lang, P. J. (2007). The International Affective Digitized Sounds (2nd Edition; IADS-2): Affective ratings of sounds and instruction manual. Technical report B3. University of Florida, Gainesville, Fl.

Bradley, M. M., Codispoti, M., Cuthbert, B. N., \& Lang, P. (2001a). Emotion and Motivation I: Defensive and Appetitive Reactions in Picture Processing. Emotion, 1(3), 276-298. doi:10.1037/1528-3542.1.3.276

Bradley, M. M., Codispoti, M., Sabatinelli, D., \& Lang, P. J. (2001b). Emotion and Motivation II: Sex Differences in Picture Processing. Emotion, 1(3), 300-319. doi:10.1037/1528-3542.1.3.300

Bradley, M.M., \& Lang, P.J. (2017). Affective Norms for English Words (ANEW): Instruction manual and affective ratings. Technical Report C-3. Gainesville, FL: UF Center for the Study of Emotion and Attention. 
Bradley, M.M., Greenwald, M., \& Hamm, A.O. (1993). Affective picture processing. In N. Birbaumer \& A. Öhman (Eds), The structure of emotion. Psychological, cognitive and clinical aspects (pp. 48-65). Seattle, WA: Hogrefe \& Huber Publishers.

Bullack, A., Büdenbender, N., Roden, I., \& Kreutz, G. (2018). Psychophysiological responses to "happy" and "sad" music: A replication study. Music Perception: An Interdisciplinary Journal, 35(4), 502-17. doi:10.1525/MP.2018.35.4.502

Carretié, L., Tapia, M., López-Martín, S., \& Albert, J. (2019). EmoMadrid: An emotional pictures database for affect research. Motivation and Emotion, 43(6), 929-939. doi:10.1007/s11031-019-09780-y

Ciuffini, R., Stratta, P., \& Marrelli, A. (2018). Emotional reactivity in mesial temporal lobe epilepsy: A pilot study. Epilepsy \& Behavior, 82, 87-90. doi:10.1016/j.yebeh.2018.02.011

Conzelmann, A., McGregor, V., \& Pauli, P. (2015). Emotion regulation of the affectmodulated startle reflex during different picture categories. Psychophysiology, 52(9), 1257-1262. doi:10.1111/psyp. 12450

Davidson, R. J. (1998). Affective style and affective disorders: Perspectives from affective neuroscience. Cognition and Emotion, 12(3), 307-330. doi:10.1080/026999398379628

Dienes, Z. (2008). Understanding psychology as a science: An Introduction to Scientific and Statistical Inference. Basingstoke, UK: Palgrave Macmillan.

Duesenberg, M., Weber, J., Schulze, L., Schaeuffele, C., Roepke, S., Hellmann-Regen, J.,...\& Wingenfeld, K. (2016). Does cortisol modulate emotion recognition and empathy? Psychoneuroendocrinology, 66, 221-227. doi:10.1016/10.1016/j.psyneuen.2016.01.011

Eerola, T., \& Vuoskoski, J. K. (2011). A comparison of the discrete and dimensional models of emotion in music. Psychology of Music, 39(1), 18-49. doi:10.1177/0305735610362821

Eerola, T., \& Vuoskoski, J. K. (2013). A review of music and emotion studies: Approaches, emotion models, and stimuli. Music Perception, 30(3), 307-340.

doi:10.1525/MP.2012.30.3.307

Eerola, T., Vuoskoski, J. K., Peltola, H-R., Putkinen, V., \& Schäfer, K. (2018). An integrative review of the enjoyment of sadness associated with music. Physics of life reviews, 25, 100121. doi:10.1016/j.plrev.2017.11.016

Ekman, P. (1992). An argument for basic emotions. Cognition and Emotion, 6(3/4), 169-200. doi:10.1080/02699939208411068

Ekman, P., \& Friesen, W. V. (1976). Pictures of facial affect. Palo Alto, California: Consulting Psychologists Press.

Evans, P., \& Schubert, E. (2008). Relationship between expressed and felt emotions in music. Musicae Scientae, 12(1), 75-79. doi:10.1177/102986490801200105 
Fernández-Abascal, E. G., Guerra, P., Martínez, F., Domínguez, F. J., Muñoz, M. A., Egea, D. A...\& Vila, J. (2008). El Sistema Internacional de Sonidos Afectivos (IADS): adaptación española. Psicothema, 20(1), 104-113.

Frieler, K., Müllensiefen, D., Fischinger, T., Schlemmer, K., Jakubowski, K., \& Lothwesen, K. (2013). Replication in music psychology. Musicae Scientiae, 17(3), 265-276. doi:10.1177/1029864913495404

Frieler, K., Müllensiefen, D., Fischinger, T., Schlemmer, K., Jakubowski, K., \& Lothwesen, K. (2013). Replication in music psychology. Musicae Scientiae, 17(3), 265-276. doi:10.1177/1029864913495404

Fritz, T., Jentschke, S., Gosselin, N., Sammler, D., Peretz, I., Turner, R.,... Koelsch, S. (2009). Universal recognition of three basic emotions in music. Current Biology, 19(7), 573-576. doi:10.1016/j.cub.2009.02.058

Fuentes-Sánchez, Jaén, I., Escrig, M.A., Lucas, I., \& Pastor, M.C. (2019). Cognitive reappraisal during unpleasant picture processing: Subjective self-report and peripheral physiology. Psychophysiology, 56(8):e13372. doi:10.1111/psyp.13372

Gabrielsson, A. (2002). Emotion perceived and emotion felt: same or different? Musicae Scientiae, 5(1 suppl), 123-47.doi:10.1177/10298649020050S105

Gomez, P., \& Danuser, B. (2004). Affective and physiological responses to environmental noises and music. International Journal of Psychophysiology, 53(2), 91-103. doi:10.1016/j.ijpsycho.2004.02.002

Gomez, P., von Gunten, A., \& Danuser, B. (2016). Autonomic nervous system reactivity within the valence-arousal affective space: Modulation by sex and age. International Journal of Psychophysiology, 109, 51-62. doi:10.1016/j.ijpsycho.2016.10.002

Gosselin, N., Peretz, I., Johnsen, E., \& Adolphs, R. (2007). Amygdala damage impairs emotion recognition from music. Neuropsychologia, 45(2), 236-244. doi:10.1016/j.neuropsychologia.2006.07.012

Gosselin, N., Peretz, I., Noulhiane, M., Hasboun, D., Beckett, C., Baulac, M., \& Samson, S. (2005). Impaired recognition of scary music following unilateral temporal lobe excision. Brain: A Journal of Neurology, 128(3), 628-640. doi:10.1093/brain/awh420

Greenwald, M. K., Cook, E.W., \& Lang, P. J. (1989). Affective judgement and psychophysiological response: Dimensional covariation in the evaluation of pictorial stimuli. Journal of Psychophysiology, 3(1), 51-64.

Grimshaw, G. M., Bulman-Fleming, M. B., \& Ngo, C. (2004). A signal-detection analysis of sex differences in the perception of emotional faces. Brain and Cognition, 54(3), 240-250. doi:10.1016/j.bandc.2004.02.029 
Hall, J. A., \& Matsumoto, D. (2004). Gender differences in judgements of multiple emotions from facial expressions. Emotion, 4(2), 201-206. doi:10.1037/1528-3542.4.2.201

Huron, D. (2011). Why is sad music pleasurable? A possible role for prolactin. Musicae Scientiae, 15(2), 146-158. doi:10.1177/1029864911401171

Imbir, K., \& Golab, M. (2017). Affective reactions to music: Norms for 120 excerpts of modern and classical music. Psychology of Music, 45(3), 432-449. doi:10.1177/0305735616671587

Juslin, P. N. (2001). Communicating emotion in music performance: A review and a theoretical framework. In P. N. Juslin \& J. A. Sloboda (Eds.), Series in affective science. Music and emotion: Theory and research (pp. 309-340). Oxford, New York: Oxford University Press.

Juslin, P. N., \& Laukka, P. (2003). Communication of emotions in vocal expression and music performance: Different channels, same code? Psychological Bulletin, 129(5), 770814. doi:10.1037/0033-2909.129.5.770

Juslin, P. N., \& Laukka, P. (2004). Expression, Perception, and Induction of Musical Emotions: A Review and a Questionnaire Study of Everyday Listening. Journal of New Music Research, 33(3), 217-238. doi:10.1080/0929821042000317813

Kallinen, K., \& Ravaja, N. (2006). Emotion perceived and emotion felt: Same and different. Musicae Scientiae, 10(2), 191-213. doi:10.1177/102986490601000203

Kreutz, G., Ott, U., Teichmann, D., Osawa, P., \& Vaitl, D. (2008). Using music to induce emotions: Influences of musical preference and absorption. Psychology of Music, 36(1), 101-26. doi:10.1177/0305735607082623

Kurdi, B., Lozano, S., \& Banaji, M. R. (2017). Introducing the Open Affective Standardized Image Set (OASIS). Behaviour research methods, 49(2), 457-470. doi:10.3758/s13428016-0715-3

Kwuon, S. J. (2009). An examination of cue redundancy theory in cross-cultural decoding of emotions in music. Journal of Music Therapy, 46(3), 217-237. doi:10.1093/jmt/46.3.217

Lang, P. J., Bradley, M. M., \& Cuthbert, B. N. (1999). International affective picture system (IAPS): Technical manual and affective ratings. Gainesville, FL: The Center for Research in Psychophysiology, University of Florida.

Laukka, P., Eerola, T., Thingujam, N.S., Yamasaki, T., \& Beller, G. (2013). Universal and culture-specific factors in the recognition and performance of musical affect expressions. Emotion, 13(3), 434-449. doi:10.1037/a0031388

Lepping, R. J., Atchley, R. A., \& Savage, C. R. (2016). Development of a validated emotionally provocative musical stimulus set for research. Psychology of Music, 44(5), 1012-1028. doi:10.1177/0305735615604509 
Marchewka, A., Zurawski, L., Jednoróg, K., \& Grabowska, A. (2014). The Nencki Affective Picture System (NAPS): introduction to a novel standardized, wide-range, high-quality, realistic picture database. Behaviour research methods, 46(2), 596-610. doi:10.3758/s13428-013-0379-1

Mesquita, B., \& Walker, R. (2003). Cultural differences in emotions: a context for interpreting emotional experiences. Behaviour Research and Therapy, 41(7), 777-793. doi:10.1016/S0005-7967(02)00189-4

Mitterschiffthaler, M. T., Fu, C. H., Dalton, J. A., Andrew, C. M., \& Williams, S. C. (2007). A functional MRI study of happy and sad affective states induced by classical music. Human Brain Mapping, 28(11), 1150-1162. doi:10.1002/hbm.20337

Moltó, J., Montañés, S., Poy, R., Segarra, P., Pastor, M. C., Tormo, M.P., ...Vila, J. (1999). Un nuevo método para el estudio experimental de las emociones: el International Affective Picture System (IAPS). Adaptación española. Revista de Psicología General y Aplicada: Revista de la Federación Española de Asociaciones de Psicología, 52(1), 55-87.

Molto, J., Segarra, P., Lopez, R., Esteller, A., Fonfria, A., Pastor, M. C., \& Poy, R. (2013). Spanish adaptation of the "International Affective Picture System" (IAPS). Third part. Anales de Psicología, 29(3), 965-984. doi:10.6018/analesps.29.3.153591

Nolen-Hoeksema, S., \& Aldao, A. (2011). Gender and age differences in emotion regulation strategies and their relationship to depressive symptoms. Personality and Individual Differences, 51(6), 704-8. doi:10.1016/j.paid.2011.06.012

Ozawa, S., Kanayama, N., \& Hiraki, K. (2019). Emotion-related cerebral blood flow changes in the ventral medial prefrontal cortex: An NIRS study. Brain and Cognition, 134, 21-28. doi:10.1016/j.bandc.2019.05.001

Paquette, S., Peretz, I., \& Belin, P. (2013). The "Musical Emotional Bursts": a validated set of musical affect bursts to investigate auditory affective processing. Frontiers in Psychology, 4. doi:10.3389/fpsyg.2013.00509

Rahman, Q., Wilson, G. D., \& Abrahams, S. (2004). Sex, sexual orientation, and identification of positive and negative facial affect. Brain and Cognition, 54(3), 179-185. doi:10.1016/j.bandc.2004.01.002

Redondo, J., Fraga, I., Padrón, I., \& Comesaña, M. (2007). The Spanish adaptation of ANEW (Affective Norms for English Words). Behaviour Research Methods, 39(3), 600-605. doi:10.3758/BF03193031

Redondo, J., Fraga, I., Padrón, I., \& Piñeiro, A. (2008). Affective ratings of sound stimuli. Behavior research methods, 40(3), 784-790. doi:10.3758/brm.40.3.784

Roy, M., Mailhot, J. P., Gosselin, N., Paquette, S., \& Peretz, I. (2009). Modulation of the startle reflex by pleasant and unpleasant music. International Journal of Psychophysiology, 71(1), 37-42. doi:10.1016/j.ijpsycho.2008.07.010 
Russell, J. A. (1980). A circumplex model of affect. Journal of Personality and Social Psychology, 39(6), 1161-1178. doi:10.1037/h0077714

Saarikallio, S., \& Erkkilä, J. (2007). The role of music in adolescents' mood regulation. Psychology of Music, 35(1), 88-109. doi:10.1177/0305735607068889

Sander, K., \& Scheich, H. (2001). Auditory perception of laughing and crying activates human amygdala regardless of attentional state. Brain Research. Cognitive Brain Research, 12(2), 181-198. doi:10.1016/s0926-6410(01)00045-3

Schimmack, U., \& Grob, A. (2000). Dimensional models of core affect: A quantitative comparison by means of structural equation modeling. European Journal of Personality, 14(4), 325-345. doi:10.1002/1099-0984(200007/08)14:4<325::AID-PER380>3.0.CO;2-I

Schimmack, U., \& Reisenzein, R. (2002). Experiencing activation: Energetic arousal and tense arousal are not mixtures of valence and activation. Emotion, 2(4), 412-417. doi:10.1037/1528-3542.2.4.412

Sege, C. T., Bradley, M. M., \& Lang, P. (2014). Startle modulation during emotional anticipation and perception. Psychophysiology, 51(10), 977-981. doi:10.1111/psyp.12244

Song, Y., Dixon, S., Pearce, M., \& Halpern, A.R. (2016). Perceived and Induced Emotion Responses to Popular Music: Categorical and Dimensional Models. Music Perception, 33(4), 472-492. doi:10.1525/mp.2016.33.4.472

Susino, M., \& Schubert, E. (2017). Cross-cultural anger communication in music: Towards a stereotype theory of emotion in music. Musicae Scientiae, 21(1), 60-74. doi:10.1177/1029864916637641

Thompson, F. W. (2015). Music, Thought, and feeling: Understanding the psychology of music. 2nd Revised Edition. New York, USA: Oxford University Press Inc.

Thompson, W. F., \& Baklwill, L-L. (2010). Cross-Cultural Similarities and Differences. In Juslin, P.N., and Sloboda, J. (Ed.), Handbook of Music and Emotion: Theory, Research, Applications (pp 755-788). Oxford, New York: Oxford University Press.

Trost, W., Ethofer, T., Zentner, M., \& Vuilleumier, P. (2012). Mapping aesthetic musical emotions in the brain. Cerebral Cortex, 22(12), 2769-2783. doi:10.1093/cercor/bhr353

Van Goethem, A., \& Sloboda, J. (2011). The functions of music for affect regulation. Musicae Scientiae, 15(2), 208-228. doi:10.1177/1029864911401174

Vieillard, S., Peretz, I., Gosselin, N., Khalfa, S., Gagnon, L., \& Bouchard, B. (2008). Happy, sad, scary and peaceful musical excerpts for research on emotions. Cognition and Emotion, 22(4), 720-752. doi:10.1080/02699930701503567

Vila, J., Sánchez, M., Ramírez, I., Fernández, M. C., Cobos, P., Rodríguez, S., .. Moltó, J. (2001). El Sistema Internacional de Imágenes Afectivas (IAPS): Adaptación española. 
Segunda parte. Revista de Psicología General y Aplicada: Revista de la Federación Española de Asociaciones de Psicología, 54(4), 635-657.

Wundt, W. (1896). Grundriss der psychologie. Leipzig, Germany: Engelmann. 


\section{FOOTNOTES}

1. The adjectives used in the instructions were the same of Eerola \& Vuoskoski (2011) study. A back translation was conducted where two bilingual experienced researchers translated from English to Spanish all adjectives. Then, a third researcher reviewed the convergence between both translations, and the most accurate version was chosen when a discrepancy was found. Finally, another different bilingual researcher translated the adjectives from English to Spanish with the aim to determine whether the output coincided with the original. The following adjectives ("felicidad", "tristeza", "ternura", "ira", "miedo") were used in the categorical model to describe discrete emotions. Regarding valence, the adjectives were “desagradable-agradable", "malo-bueno", "negativo-positivo". For energy arousal the adjectives were "adormilado-despierto", “somnoliento-alerta", "cansado-desvelado". Finally, the adjectives used for tension arousal were "relajado-excitado", "calmado-en tensión", “tranquilo-nervioso". For the English version of adjectives, see Eerola \& Vuoskoski (2011). 


\section{FIGURE CAPTION}

Figure 1. Mean ratings of affective dimensions and discrete emotions for all excerpts $(\mathrm{N}=$ 102) for Spanish and Finnish population. The marker types represent the target emotion categories. 

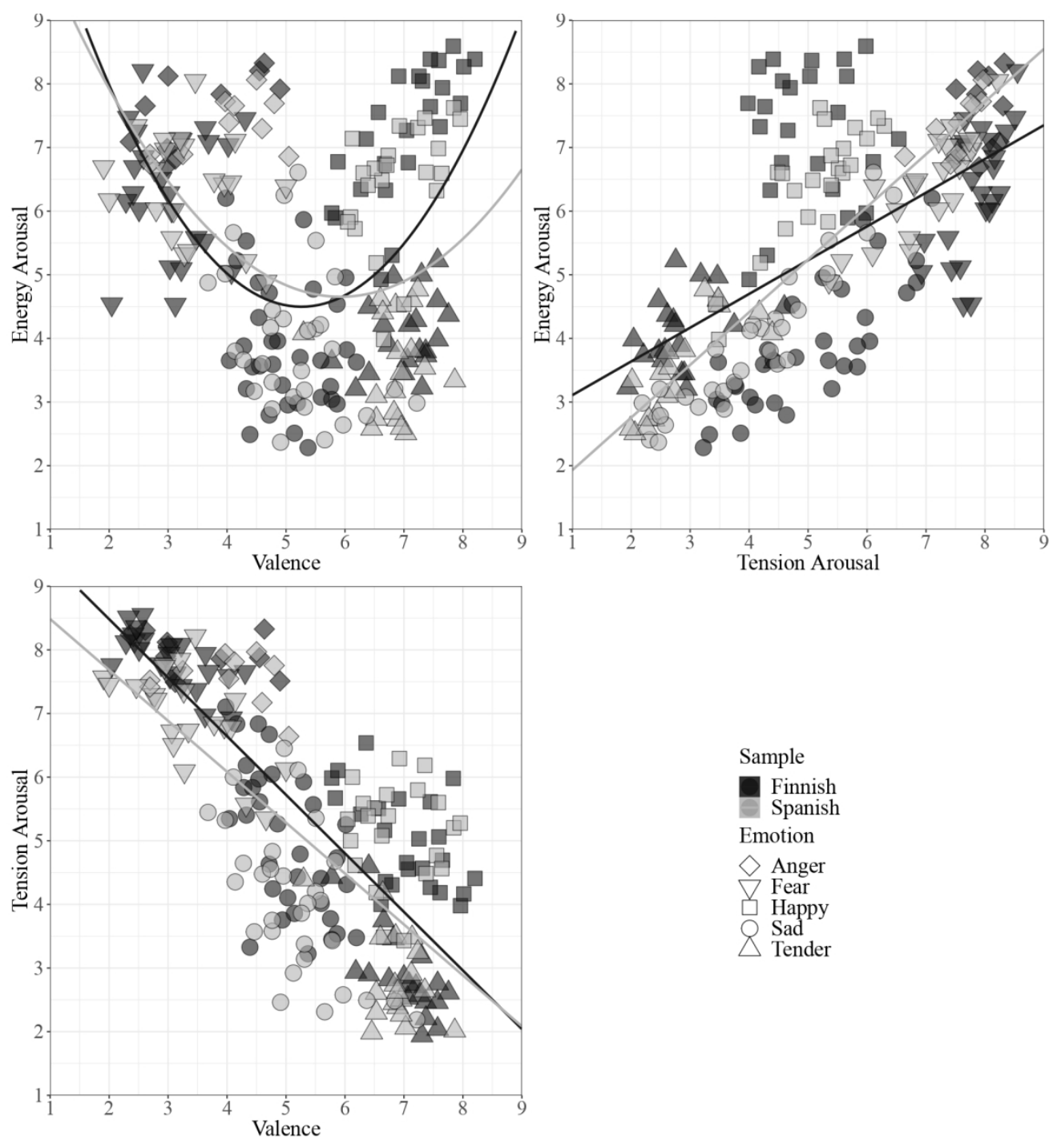

Figure 1. Mean ratings of affective dimensions and discrete emotions for all excerpts $(\mathrm{N}=102)$ for Spanish and Finnish population. The marker types represent the target emotion categories. 
Table 1. Mean (M) and Standard Deviation (SD) for each scale in the overall Finnish sample, as well as set 1 and set 2, separately (these data correspond to the study by Eerola \& Vuoskoski (2011).

\begin{tabular}{|c|c|c|c|c|c|c|}
\hline & \multicolumn{2}{|c|}{ GLOBAL } & \multicolumn{2}{|c|}{ SET 1} & \multicolumn{2}{|c|}{ SET 2} \\
\hline & $M$ & SD & $M$ & SD & $M$ & SD \\
\hline \multicolumn{7}{|c|}{ Categorical Model } \\
\hline Anger & 1.99 & 1.52 & 1.98 & 1.46 & 2.00 & 1.60 \\
\hline Fear & 2.78 & 1.89 & 2.85 & 1.96 & 2.70 & 1.83 \\
\hline Happiness & 2.58 & 1.89 & 2.66 & 1.92 & 2.49 & 1.89 \\
\hline Tenderness & 2.63 & 1.58 & 2.65 & 1.59 & 2.60 & 1.58 \\
\hline Sadness & 2.98 & 1.64 & 2.88 & 1.66 & 3.08 & 1.63 \\
\hline \multicolumn{7}{|c|}{ Dimensional Model } \\
\hline Valence & 5.28 & 1.73 & 5.23 & 1.77 & 5.33 & 1.71 \\
\hline Energy & 5.47 & 1.81 & 5.57 & 1.93 & 5.37 & 1.71 \\
\hline Tension & 5.45 & 1.98 & 5.49 & 2.04 & 5.41 & 1.94 \\
\hline
\end{tabular}


Table 2. Descriptive statistics (M, SD, confidence interval; $\mathrm{Cl}$ ) for affective ratings of 102 musical excerpts for men and women separately, besides $t$ test between both sex and effect size (d).

\begin{tabular}{|c|c|c|c|c|c|c|c|c|}
\hline & \multicolumn{3}{|c|}{ Men } & \multicolumn{3}{|c|}{ Women } & \multirow[b]{3}{*}{$t$} & \multirow[b]{3}{*}{$d$} \\
\hline & \multirow[b]{2}{*}{$M(S D)$} & \multicolumn{2}{|c|}{$95 \% \mathrm{Cl}$} & \multirow[b]{2}{*}{$M(S D)$} & \multicolumn{2}{|c|}{$95 \% \mathrm{Cl}$} & & \\
\hline & & Lower & Upper & & Lower & Upper & & \\
\hline \multicolumn{9}{|l|}{ Categorical Model } \\
\hline Happiness & $3.63(1.95)$ & 3.25 & 4.02 & $3.51(2.13)$ & 3.09 & 3.93 & $t(101)=2.11, p=.019$ & .06 \\
\hline Anger & $2.79(1.83)$ & 2.43 & 3.15 & $2.91(2.01)$ & 2.52 & 3.31 & $t(101)=2.50, p=.007$ & .06 \\
\hline Fear & $3.37(1.97)$ & 2.98 & 3.75 & $3.53(2.27)$ & 3.09 & 3.98 & $t(101)=3.34, p=.001$ & .08 \\
\hline Tenderness & $3.84(2.02)$ & 3.45 & 4.24 & $3.49(2.00)$ & 3.09 & 3.88 & $t(101)=7.11, p<.001$ & .17 \\
\hline Sadness & $3.93(1.54)$ & 3.62 & 4.23 & $3.83(1.72)$ & 3.49 & 4.16 & $t(101)=1.67, p=.049$ & .06 \\
\hline \multicolumn{9}{|l|}{ Dimensional Model } \\
\hline Valence & $5.51(1.40)$ & 5.24 & 5.79 & $5.30(1.65)$ & 4.98 & 5.62 & $t(101)=4.47, p<.001$ & .14 \\
\hline Energy Arousal & 5.09 (1.59) & 4.78 & 5.41 & $5.30(1.75)$ & 4.95 & 5.64 & $t(101)=4.62, p<.001$ & .13 \\
\hline Tension & $4.90(1.79)$ & 4.55 & 5.25 & $5.04(1.87)$ & 4.67 & 5.50 & $t(101)=2.80, p=.003$ & .08 \\
\hline
\end{tabular}


Table 3. Pairwise correlations between emotion concepts.

\begin{tabular}{|c|c|c|c|c|c|c|c|}
\hline & Happiness & Sadness & Tenderness & Fear & Anger & Valence & Energy Arousal \\
\hline Sadness & $-.45^{* * *}$ & & & & & & \\
\hline Tenderness & $.57 * * *$ & $.34 * * *$ & & & & & \\
\hline Fear & $-.80 * * *$ & -.11 & $-.83 * * *$ & & & & \\
\hline Anger & $-.68 * * *$ & $-.23^{*}$ & $-.83 * * *$ & $.86 * * *$ & & & \\
\hline Valence & $.87^{* * *}$ & -.06 & $.81 * * *$ & $-.95 * * *$ & $-.82 * * *$ & & \\
\hline Energy Arousal & .06 & $-.80 * * *$ & $-.70 * * *$ & $.42 * * *$ & $.61^{* * *}$ & $-.31 * *$ & \\
\hline Tension Arousal & $-.36 * * *$ & $-.58 * * *$ & $-.90 * * *$ & $.76 * * *$ & $.84 * * *$ & $-.68 * * *$ & $.89 * * *$ \\
\hline
\end{tabular}

Note: ${ }^{* * *} p<0.001 ;{ }^{* *} p<0.01 ;{ }^{*} p<0.05$ 
Table 4. Partial correlations between affective ratings of basic emotions.

\begin{tabular}{lclll}
\hline & Happiness & Sadness & Tender & Fear \\
\hline Sadness & $-.91^{* * *}$ & & & \\
Tenderness & $.21^{* * *}$ & $.35^{* * *}$ & & \\
Fear & $-.73^{* * *}$ & $.57^{* *}$ & $-.18^{* * *}$ & \\
Anger & $-.42^{* * *}$ & $-.43^{*}$ & $-.18^{* * *}$ & .05 \\
\hline
\end{tabular}

Note: ${ }^{* * *} p<0.001 ;{ }^{* *} p<0.01 ;{ }^{*} p<0.05$ 
Table 5. Summary of fit $\left(R^{2}\right)$ from regression analysis for Spanish normative ratings.

\begin{tabular}{ll}
\hline & \multicolumn{1}{c}{ Spanish data } \\
\hline Dimensions as predictors (valence, energy, tension) & \\
Happiness & $.89\left(\mathrm{~V}_{0.83}, \mathrm{E}_{1.17}, \mathrm{~T}_{-0.89}\right)$ \\
Sadness & $.73\left(\mathrm{~V}_{-0.50}, \mathrm{E}_{-0.65}, \mathrm{~T}_{-0.27}\right)$ \\
Tenderness & $.88\left(\mathrm{~V}_{0.41}, \mathrm{E}_{0.07}, \mathrm{~T}_{-0.80}\right)$ \\
Fear & $.92\left(\mathrm{~V}_{-0.74}, \mathrm{E}_{-0.62}, \mathrm{~T}_{0.99}\right)$ \\
Anger & $.82\left(\mathrm{~V}_{-0.47}, \mathrm{E}_{-0.17}, \mathrm{~T}_{0.77}\right)$ \\
Mean $R^{2}$ & .85 \\
Discrete emotions as predictors (happiness, sadness, tenderness, fear, anger) & \\
Valence & $.94\left(\mathrm{H}_{0.28}, \mathrm{~S}_{-0.01}, \mathrm{~T}_{0.15}, \mathrm{~F}_{-0.38}, \mathrm{~A}_{0.04}\right)$ \\
$\quad$ Energy & $.92\left(\mathrm{H}_{0.61}, \mathrm{~S}_{-0.13}, \mathrm{~T}_{-0.49}, \mathrm{~F}_{-0.001}, \mathrm{~A}_{0.53}\right)$ \\
Tension & $.94\left(\mathrm{H}_{0.28}, \mathrm{~S}_{-0.17}, \mathrm{~T}_{-0.47}, \mathrm{~F}_{0.17}, \mathrm{~A}_{0.39}\right)$ \\
Mean $R^{2}$ & .93 \\
\hline
\end{tabular}


Table 6. Descriptive statistics (Mean, Standard deviation, $\mathrm{Cl}$ ) of affective ratings of 102 musical excerpts, separately for Spanish and Finnish population, besides $t$ test between both groups and effect size (d).

\begin{tabular}{|c|c|c|c|c|c|c|c|c|}
\hline & \multicolumn{3}{|c|}{ Spanish } & \multicolumn{3}{|c|}{ Finnish } & \multirow[b]{3}{*}{$t$} & \multirow[b]{3}{*}{$d$} \\
\hline & \multirow[b]{2}{*}{$M(S D)$} & \multicolumn{2}{|c|}{$95 \% \mathrm{Cl}$} & \multirow[b]{2}{*}{$M(S D)$} & \multicolumn{2}{|c|}{$95 \% \mathrm{Cl}$} & & \\
\hline & & Lower & Upper & & Lower & Upper & & \\
\hline \multicolumn{9}{|c|}{ Categorical Model } \\
\hline Happiness & $3.54(2.06)$ & 3.14 & 3.95 & $2.58(1.90)$ & 2.21 & 2.95 & $t(101)=14.32, p<.0001$ & .48 \\
\hline Anger & $2.88(1.95)$ & 2.49 & 3.26 & $1.99(1.52)$ & 1.69 & 2.29 & $t(101)=12.23, p<.0001$ & .51 \\
\hline Fear & $3.48(2.18)$ & 3.06 & 3.91 & $2.78(1.89)$ & 2.41 & 3.15 & $t(101)=10.97, p<.0001$ & .34 \\
\hline Tenderness & $3.58(2.00)$ & 3.19 & 3.98 & $2.63(1.58)$ & 2.32 & 2.94 & $t(101)=12.15, p<.0001$ & .53 \\
\hline Sadness & $3.85(1.65)$ & 3.53 & 4.18 & $2.98(1.64)$ & 2.66 & 3.30 & $t(101)=12.91, p<.0001$ & .53 \\
\hline \multicolumn{9}{|c|}{ Dimensional Model } \\
\hline Valence & $5.36(1.57)$ & 5.05 & 5.67 & $5.28(1.73)$ & 4.94 & 5.62 & $t(101)=1.55, p=.062$ & .05 \\
\hline Energy & $5.24(1.70)$ & 4.90 & 5.57 & $5.47(1.81)$ & 5.11 & 5.83 & $t(101)=3.71, p=.0002$ & .13 \\
\hline Tension & $5.00(1.83)$ & 4.64 & 5.36 & $5.45(1.98)$ & 5.06 & 5.84 & $t(101)=6.39, p<.0001$ & .24 \\
\hline
\end{tabular}


Appendix (Table 1). Mean (M) and Standard Deviation (SD) in the different affective scales for each excerpt for the Spanish sample $(n=129)$

\begin{tabular}{|c|c|c|c|c|c|c|c|c|c|c|c|c|c|c|c|c|c|c|}
\hline \multirow[b]{2}{*}{ № } & \multirow[b]{2}{*}{$\begin{array}{c}\text { Soundtrack } \\
\text { title }\end{array}$} & \multirow[b]{2}{*}{ Duration } & \multicolumn{2}{|c|}{ Happiness } & \multicolumn{2}{|c|}{ Sadness } & \multicolumn{2}{|c|}{ Tenderness } & \multicolumn{2}{|c|}{ Fear } & \multicolumn{2}{|c|}{ Anger } & \multicolumn{2}{|c|}{ Valence } & \multicolumn{2}{|c|}{$\begin{array}{l}\text { Energy } \\
\text { Arousal }\end{array}$} & \multicolumn{2}{|c|}{$\begin{array}{l}\text { Tension } \\
\text { Arousal }\end{array}$} \\
\hline & & & M & SD & M & SD & $\mathrm{M}$ & SD & $\mathrm{M}$ & SD & $\mathrm{M}$ & SD & M & SD & M & SD & $\mathrm{M}$ & SD \\
\hline 001 & Shallow Grave & 15 & 8.23 & 1.01 & 1.11 & 0.53 & 3.46 & 2.09 & 1.11 & 0.40 & 1.09 & 0.29 & 7.84 & 1.13 & 7.63 & 0.98 & 5.20 & 2.09 \\
\hline 003 & The Rainmaker & 18 & 7.31 & 1.01 & 1.37 & 0.82 & 4.2 & 2.14 & 1.14 & 0.39 & 1.15 & 0.51 & 7.38 & 1.08 & 6.61 & 1.23 & 4.48 & 1.92 \\
\hline 004 & $\begin{array}{c}\text { Man of Galilee } \\
\text { CD1 }\end{array}$ & 16 & 7.63 & 1.39 & 1.18 & 0.61 & 2.95 & 1.71 & 1.23 & 0.70 & 1.43 & 1.03 & 7.23 & 1.42 & 7.31 & 1.15 & 5.90 & 1.95 \\
\hline 010 & $\begin{array}{l}\text { The } \\
\text { Untouchables }\end{array}$ & 15 & 7.89 & 1.07 & 1.47 & 0.96 & 4.36 & 2.31 & 1.17 & 0.52 & 1.17 & 0.42 & 7.58 & 1.24 & 6.98 & 1.47 & 5.6 & 2.00 \\
\hline 011 & Shine & 16 & 6.72 & 1.93 & 1.57 & 1.25 & 4.11 & 2.39 & 1.51 & 1.05 & 1.25 & 0.69 & 6.73 & 1.20 & 6.88 & 1.48 & 5.39 & 1.80 \\
\hline 016 & Big Fish & 22 & 5.12 & 2.10 & 4.26 & 2.53 & 6.43 & 2.11 & 1.22 & 0.65 & 1.14 & 0.58 & 6.89 & 1.14 & 3.78 & 1.50 & 2.63 & 1.35 \\
\hline 018 & Shine & 19 & 6.59 & 1.71 & 2.22 & 1.69 & 4.66 & 2.30 & 1.94 & 1.56 & 1.78 & 1.30 & 6.71 & 1.52 & 6.72 & 1.38 & 5.72 & 1.91 \\
\hline 020 & Vertigo OST & 15 & 6.06 & 2.01 & 2.68 & 1.86 & 4.09 & 2.21 & 1.66 & 1.11 & 1.57 & 1.03 & 6.39 & 1.47 & 6.41 & 1.26 & 5.39 & 1.63 \\
\hline 021 & Gladiator & 13 & 5.63 & 2.09 & 2.57 & 1.82 & 4.55 & 2.24 & 1.499 & 1.05 & 1.34 & 0.89 & 6.53 & 1.54 & 5.19 & 1.53 & 4.19 & 1.96 \\
\hline 022 & $\begin{array}{c}\text { Shakespeare in } \\
\text { Love }\end{array}$ & 18 & 5.67 & 2.24 & 3.41 & 2.23 & 3.14 & 1.98 & 2.23 & 1.72 & 2.19 & 1.75 & 6.31 & 1.53 & 6.60 & 1.60 & 5.58 & 1.64 \\
\hline 029 & Vertigo OST & 15 & 3.28 & 1.97 & 5.75 & 2.18 & 4.88 & 2.50 & 2.38 & 1.73 & 2.02 & 1.64 & 5.58 & 1.84 & 4.22 & 1.89 & 4.06 & 1.98 \\
\hline 030 & $\begin{array}{c}\text { The English } \\
\text { Patient }\end{array}$ & 31 & 4.4 & 1.92 & 5.66 & 2.33 & 6.72 & 1.75 & 2.08 & 1.53 & 1.29 & 0.80 & 7.22 & 1.36 & 2.98 & 1.49 & 2.19 & 1.37 \\
\hline 032 & $\begin{array}{l}\text { The English } \\
\text { Patient }\end{array}$ & 25 & 1.74 & 1.30 & 7.62 & 1.40 & 4.91 & 2.75 & 1.98 & 1.72 & 1.4 & 1.08 & 5.13 & 1.91 & 3.08 & 1.46 & 2.92 & 1.57 \\
\hline 036 & $\begin{array}{l}\text { Running } \\
\text { Scared }\end{array}$ & 21 & 1.53 & 0.98 & 5.88 & 1.91 & 2.31 & 2.09 & 5.13 & 2.00 & 3.72 & 2.10 & 3.68 & 1.87 & 4.88 & 2.15 & 5.45 & 2.20 \\
\hline 037 & $\begin{array}{l}\text { Road To } \\
\text { Perdition }\end{array}$ & 25 & 1.61 & 1.11 & 6.44 & 2.07 & 2.78 & 2.12 & 3.98 & 2.18 & 3.09 & 2.30 & 4.14 & 1.85 & 3.80 & 1.99 & 4.35 & 2.04 \\
\hline
\end{tabular}




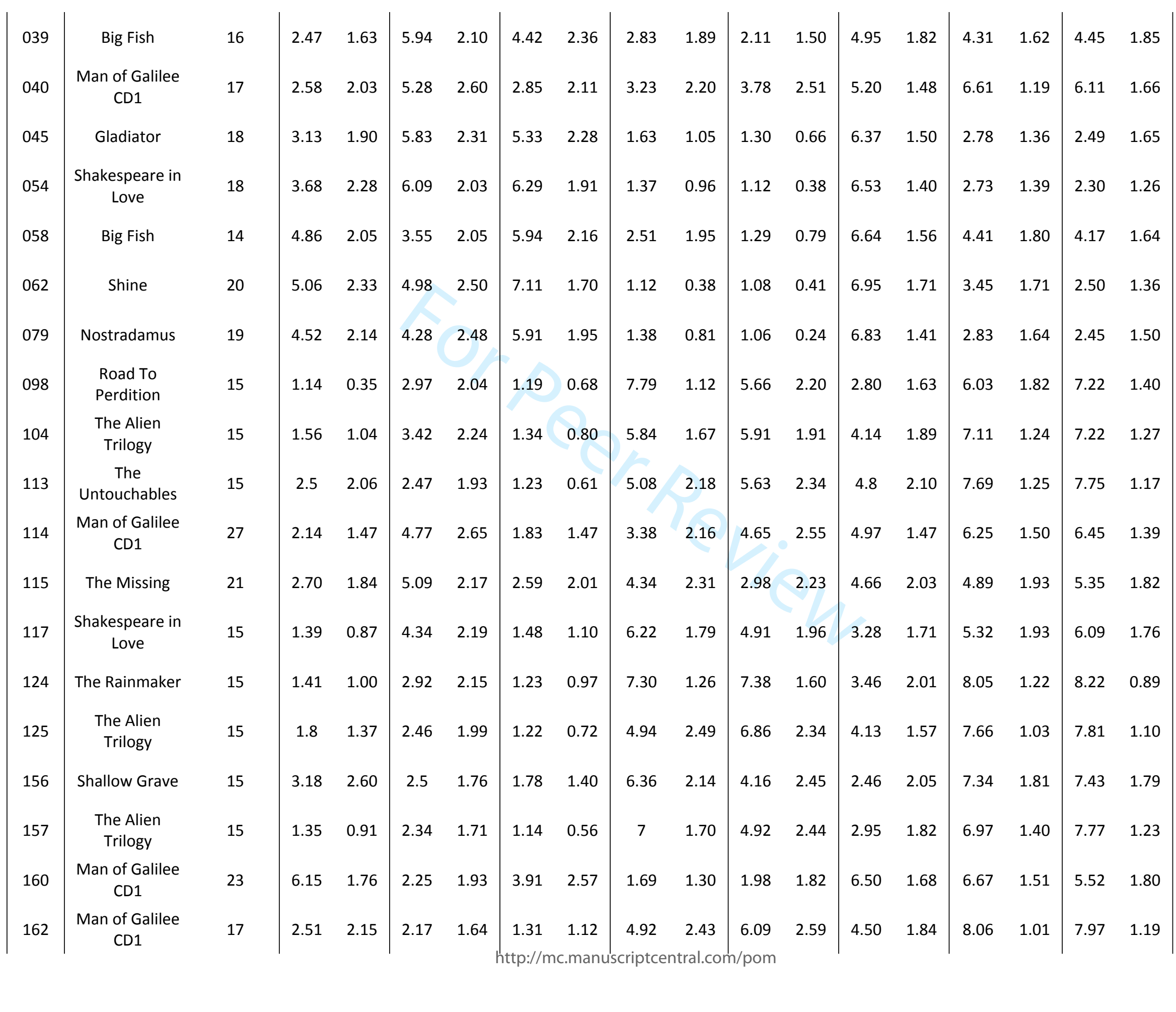




\begin{tabular}{|c|c|c|c|c|c|c|c|c|c|c|c|c|c|c|c|c|c|c|c|}
\hline $\begin{array}{l}1 \\
2\end{array}$ & 163 & Naked Lunch & 19 & 2.31 & 1.59 & 4.69 & 2.26 & 2.1 & 2.06 & 4.27 & 2.28 & 2.44 & 1.65 & 4.74 & 1.57 & 4.17 & 1.99 & 4.55 & 2.04 \\
\hline $\begin{array}{l}3 \\
4\end{array}$ & 168 & Cape Fear & 15 & 1.45 & 1.06 & 3.18 & 2.09 & 1.31 & 0.88 & 6.17 & 2.15 & 6.55 & 2.64 & 3.25 & 1.37 & 6.89 & 1.71 & 7.67 & 1.11 \\
\hline $\begin{array}{l}5 \\
6\end{array}$ & 170 & $\begin{array}{c}\text { The English } \\
\text { Patient }\end{array}$ & 22 & 1.43 & 1.02 & 3.08 & 1.85 & 1.2 & 0.59 & 7.09 & 1.44 & 5.51 & 2.28 & 3.27 & 1.52 & 7.03 & 1.23 & 7.36 & 1.28 \\
\hline $\begin{array}{l}7 \\
8 \\
9\end{array}$ & 173 & $\begin{array}{l}\text { Running } \\
\text { Scared }\end{array}$ & 14 & 3.14 & 1.97 & 2.6 & 1.95 & 1.38 & 0.84 & 3.89 & 2.41 & 3.06 & 2.18 & 5 & 1.66 & 6.39 & 1.60 & 6.13 & 1.63 \\
\hline $\begin{array}{l}10 \\
11\end{array}$ & 177 & Cape Fear & 15 & 1.30 & 0.83 & 3.72 & 2.09 & 1.5 & 1.01 & 6.73 & 1.75 & 4.44 & 2.32 & 3.09 & 1.60 & 6.02 & 1.77 & 6.51 & 1.77 \\
\hline $\begin{array}{l}12 \\
13\end{array}$ & 178 & Big Fish & 15 & 1.63 & 1.16 & 4.70 & 2.24 & 1.98 & 1.66 & 5.36 & 2.01 & 4.06 & 2.23 & 4.32 & 1.57 & 5.22 & 1.82 & 5.57 & 1.96 \\
\hline $\begin{array}{l}14 \\
15 \\
16\end{array}$ & 180 & $\begin{array}{c}\text { Shakespeare in } \\
\text { Love }\end{array}$ & 19 & 3.64 & 2.06 & 4.44 & 2.22 & 3.98 & 2.37 & 2.97 & 1.80 & 2.47 & 1.93 & 5.83 & 1.70 & 4.97 & 1.85 & 4.68 & 1.93 \\
\hline $\begin{array}{l}17 \\
18\end{array}$ & 187 & Juha & 18 & 6.48 & 1.79 & 2.86 & 1.93 & 6.77 & 1.86 & 1.19 & 0.53 & 1.03 & 0.17 & 7.23 & 1.09 & 4.77 & 1.55 & 3.25 & 1.58 \\
\hline $\begin{array}{l}19 \\
20 \\
21\end{array}$ & 188 & $\begin{array}{c}\text { Dances with } \\
\text { Wolves }\end{array}$ & 18 & 6.46 & 2.04 & 1.74 & 1.16 & 3.25 & 1.94 & 1.51 & 0.94 & 1.72 & 1.41 & 6.63 & 1.23 & 6.48 & 1.21 & 6.08 & 1.80 \\
\hline $\begin{array}{l}22 \\
23\end{array}$ & 189 & Blanc & 15 & 4.5 & 2.04 & 4.91 & 2.33 & 5.97 & 2.29 & 1.86 & 1.49 & 1.53 & 1.21 & 6.6 & 1.52 & 4.62 & 1.54 & 3.48 & 1.54 \\
\hline $\begin{array}{l}24 \\
25\end{array}$ & 190 & $\begin{array}{c}\text { Pride \& } \\
\text { Prejudice }\end{array}$ & 20 & 3.08 & 2.10 & 6.72 & 1.96 & 6.52 & 2.13 & 1.67 & 1.31 & 1.53 & 1.34 & 6.85 & 2.02 & 3.17 & 1.80 & 2.74 & 1.82 \\
\hline $\begin{array}{l}26 \\
27 \\
28\end{array}$ & 191 & The Godfarher & 16 & 5.53 & 1.83 & 4.05 & 2.32 & 6.91 & 1.81 & 1.34 & 0.80 & 1.16 & 0.51 & 7.12 & 1.38 & 3.82 & 1.78 & 2.91 & 1.53 \\
\hline $\begin{array}{l}29 \\
30\end{array}$ & 192 & Oliwer Twist & 17 & 6.97 & 1.54 & 2.06 & 1.50 & 5.13 & 2.09 & 1.25 & 0.64 & 1.23 & 0.66 & 7.55 & 1.10 & 6.32 & 1.61 & 4.77 & 1.62 \\
\hline $\begin{array}{l}31 \\
32\end{array}$ & 204 & Outbreak & 15 & 4.89 & 2.09 & 2.26 & 1.76 & 3.85 & 2.48 & 2.26 & 1.56 & 1.82 & 1.40 & 6.09 & 1.54 & $5 . .91$ & 1.52 & 5.00 & 1.78 \\
\hline $\begin{array}{l}33 \\
34 \\
35\end{array}$ & 210 & $\begin{array}{l}\text { Crouching } \\
\text { Tiger }\end{array}$ & 18 & 2.81 & 2.06 & 5.30 & 2.31 & 3.5 & 2.37 & 2.64 & 1.72 & 2.42 & 1.88 & 5.51 & 1.54 & 5.54 & 1.77 & 5.35 & 1.76 \\
\hline $\begin{array}{l}36 \\
37\end{array}$ & 215 & Grizzle Man & 27 & 1.12 & 0.41 & 2.8 & 2.35 & 1.06 & 0.24 & 7.75 & 1.53 & 3.6 & 2.50 & 2.00 & 1.32 & 6.17 & 2.10 & 7.45 & 1.70 \\
\hline $\begin{array}{l}38 \\
39\end{array}$ & 218 & Hellraiser & 15 & 1.16 & 0.44 & 3.48 & 2.13 & 1.06 & 0.24 & 7.13 & 1.41 & 7.14 & 1.98 & 2.69 & 1.75 & 6.89 & 1.77 & 7.52 & 1.49 \\
\hline $\begin{array}{l}40 \\
41 \\
42\end{array}$ & 219 & $\begin{array}{l}\text { Batman } \\
\text { Returns }\end{array}$ & 16 & 1.19 & 0.5 & 3.11 & 2.13 & 1.16 & 0.48 & 7.91 & 1.33 & 5.44 & 2.45 & 1.91 & 1.22 & 6.69 & 1.88 & 7.57 & 1.42 \\
\hline $\begin{array}{l}43 \\
44\end{array}$ & 227 & $\begin{array}{c}\text { Lethal weapon } \\
3\end{array}$ & 14 & 1.5 & 1.14 & 2.97 & 2.17 & 1.13 & 0.49 & 6.86 & 1.62 & 7.47 & 1.46 & 3.97 & 1.97 & 7.72 & 1.47 & 7.94 & 1.07 \\
\hline
\end{tabular}




\begin{tabular}{|c|c|c|c|c|c|c|c|c|c|c|c|c|c|c|c|c|c|c|}
\hline 278 & $\begin{array}{l}\text { Pride \& } \\
\text { Prejudice }\end{array}$ & 18 & 1.77 & 1.31 & 7.88 & 1.33 & 5.33 & 2.51 & 1.97 & 1.36 & 1.55 & 1.23 & 4.91 & 2.35 & 2.37 & 1.68 & 2.46 & 1.79 \\
\hline 280 & Blanc & 15 & 1.85 & 1.70 & 5.75 & 2.26 & 2.98 & 2.22 & 4.42 & 2.58 & 2.15 & 1.80 & 4.77 & 1.38 & 3.31 & 1.76 & 3.75 & 2.12 \\
\hline 283 & Blanc & 18 & 2.08 & 1.61 & 6.41 & 1.89 & 3.33 & 2.96 & 3.45 & 2.26 & 2.03 & 1.53 & 4.46 & 1.75 & 3.17 & 1.87 & 3.57 & 2.15 \\
\hline 288 & $\begin{array}{c}\text { The Portait of } \\
\text { a Lady }\end{array}$ & 22 & 1.63 & 1.28 & 8.14 & 1.03 & 4.55 & 2.51 & 2.00 & 1.62 & 1.38 & 0.82 & 5.66 & 2.13 & 2.41 & 1.22 & 2.31 & 1.30 \\
\hline 292 & Batman & 19 & 2.85 & 1.97 & 6.00 & 2.12 & 5.45 & 2.37 & 2.18 & 1.62 & 1.31 & 0.83 & 5.97 & 1.38 & 2.64 & 1.31 & 2.58 & 1.42 \\
\hline 293 & $\begin{array}{l}\text { Crouching } \\
\text { Tiger }\end{array}$ & 18 & 2.22 & 1.64 & 6.67 & 1.98 & 4.44 & 2.29 & 2.31 & 1.72 & 1.86 & 1.42 & 5.26 & 2.22 & 3.49 & 1.71 & 3.86 & 1.89 \\
\hline 294 & Juha & 15 & 2.97 & 1.82 & 5.97 & 2.10 & 4.45 & 2.25 & 1.83 & 1.16 & 1.59 & 1.09 & 5.78 & 1.65 & 3.83 & 1.64 & 3.43 & 1.62 \\
\hline 295 & Blanc & 16 & 1.68 & 1.31 & 6.37 & 2.13 & 3.46 & 2.28 & 3.52 & 2.59 & 1.57 & 1.31 & 5.31 & 1.57 & 3.19 & 1.74 & 3.38 & 1.92 \\
\hline 296 & Oliwer Twist & 29 & 4.69 & 2.08 & 5.31 & 2.12 & 6.45 & 1.95 & 1.52 & 1.12 & 1.17 & 0.49 & 6.84 & 1.24 & 3.20 & 1.41 & 2.48 & 1.25 \\
\hline 297 & Batman & 14 & 3.08 & 1.97 & 3.82 & 2.01 & 4.38 & 2.19 & 3.55 & 2.16 & 2.03 & 1.76 & 5.50 & 1.59 & 4.16 & 1.75 & 4.20 & 2.00 \\
\hline 299 & Oliwer Twist & 16 & 4.80 & 2.06 & 3.94 & 2.11 & 6.45 & 1.83 & 1.58 & 1.12 & 1.18 & 0.56 & 6.53 & 1.53 & 3.09 & 1.55 & 2.61 & 1.35 \\
\hline 306 & Hellraiser & 16 & 1.11 & 0.31 & 3.12 & 2.20 & 1.15 & 0.51 & 7.48 & 1.40 & 5.63 & 2.55 & 2.94 & 1.53 & 7.13 & 1.35 & 7.73 & 1.10 \\
\hline 309 & $\begin{array}{l}\text { The Fifth } \\
\text { Element }\end{array}$ & 14 & 1.19 & 0.53 & 3.39 & 2.22 & 1.14 & 0.66 & 6.42 & 1.82 & 7.31 & 1.47 & 2.69 & 1.78 & 6.74 & 1.81 & 7.42 & 1.51 \\
\hline 313 & Hannibal & 14 & 1.20 & 0.64 & 3.22 & 2.25 & 1.22 & 0.74 & 7.38 & 2.03 & 3.35 & 2.27 & 3.06 & 1.67 & 5.58 & 2.16 & 6.72 & 1.89 \\
\hline 316 & $\begin{array}{l}\text { Batman } \\
\text { Returns }\end{array}$ & 15 & 2.57 & 2.08 & 2.08 & 1.51 & 1.40 & 0.93 & 4.31 & 2.24 & 5.25 & 2.68 & 4.59 & 1.42 & 7.30 & 1.14 & 7.17 & 1.32 \\
\hline 320 & JFK & 17 & 1.51 & 1.06 & 2.32 & 1.87 & 1.28 & 0.80 & 6.52 & 2.10 & 4.89 & 2.65 & 4.06 & 1.46 & 6.45 & 1.39 & 6.78 & 1.44 \\
\hline 321 & $\begin{array}{l}\text { The Fifth } \\
\text { Element }\end{array}$ & 19 & 1.58 & 1.37 & 2.40 & 1.81 & 1.28 & 0.84 & 5.65 & 2.25 & 6.22 & 2.18 & 3.78 & 1.52 & 6.48 & 1.43 & 6.84 & 1.45 \\
\hline 325 & $\begin{array}{l}\text { Lethal weapon } \\
\qquad 3\end{array}$ & 16 & 2.16 & 1.73 & 4.05 & 2.33 & 2.19 & 1.84 & 5.09 & 2.07 & 3.52 & 2.35 & 4.11 & 1.53 & 5.66 & 1.81 & 6.00 & 1.70 \\
\hline 329 & Oliwer Twist & 16 & 2.11 & 1.47 & 4.95 & 2.05 & 2.45 & 1.86 & 5.25 & 1.90 & 3.03 & 2.03 & 4.77 & 1.69 & 4.45 & 1.94 & 4.83 & 1.97 \\
\hline
\end{tabular}




\begin{tabular}{|c|c|c|c|c|c|c|c|c|c|c|c|c|c|c|c|c|c|c|}
\hline 331 & $\begin{array}{l}\text { Pride \& } \\
\text { Prejudice }\end{array}$ & 14 & 4.00 & 2.36 & 5.34 & 2.31 & 7.12 & 1.77 & 1.37 & 0.89 & 1.11 & 0.36 & 6.45 & 1.81 & 2.58 & 1.38 & 1.98 & 1.24 \\
\hline 333 & $\begin{array}{l}\text { Lethal weapon } \\
3\end{array}$ & 18 & 5.13 & 2.25 & 4.06 & 2.43 & 7.02 & 1.87 & 1.13 & 0.42 & 1.13 & 0.55 & 7.35 & 1.16 & 3.54 & 1.57 & 2.65 & 1.53 \\
\hline 335 & $\begin{array}{l}\text { Pride \& } \\
\text { Prejudice }\end{array}$ & 16 & 4.25 & 2.28 & 5.09 & 2.56 & 7.31 & 1.74 & 1.31 & 1.08 & 1.08 & 0.32 & 7.03 & 1.30 & 2.51 & 1.24 & 2.06 & 1.12 \\
\hline 343 & Gizzly Man & 27 & 4.80 & 2.12 & 5.06 & 2.53 & 7.63 & 1.53 & 1.09 & 0.43 & 1.03 & 0.18 & 7.86 & 1.34 & 3.34 & 1.67 & 2.02 & 1.27 \\
\hline 346 & Oliwer Twist & 17 & 5.55 & 2.08 & 3.38 & 2.28 & 5.65 & 2.09 & 1.20 & 0.51 & 1.05 & 0.21 & 6.86 & 1.19 & 4.52 & 1.63 & 3.50 & 1.73 \\
\hline 357 & Dracula & 14 & 3.57 & 2.14 & 4.00 & 2.33 & 5.29 & 2.54 & 3.45 & 2.52 & 1.54 & 1.40 & 5.30 & 1.86 & 4.08 & 2.25 & 4.39 & 2.08 \\
\hline 360 & Batman & 19 & 1.98 & 1.42 & 4.55 & 2.22 & 2.28 & 1.69 & 6.11 & 1.95 & 2.95 & 2.15 & 4.60 & 1.85 & 3.60 & 1.97 & 4.48 & 2.14 \\
\hline
\end{tabular}


Appendix (Table 2). Mean (M) and Standard Deviation (SD) in the different affective scales for each excerpt for Spanish women ( $n=92$ )

\begin{tabular}{|c|c|c|c|c|c|c|c|c|c|c|c|c|c|c|c|c|c|c|}
\hline \multirow[b]{2}{*}{ № } & \multirow[b]{2}{*}{$\begin{array}{c}\text { Soundtrack } \\
\text { title }\end{array}$} & \multirow[b]{2}{*}{ Duration } & \multicolumn{2}{|c|}{ Happiness } & \multicolumn{2}{|c|}{ Sadness } & \multicolumn{2}{|c|}{ Tenderness } & \multicolumn{2}{|c|}{ Fear } & \multicolumn{2}{|c|}{ Anger } & \multicolumn{2}{|c|}{ Valence } & \multicolumn{2}{|c|}{$\begin{array}{l}\text { Energy } \\
\text { Arousal }\end{array}$} & \multicolumn{2}{|c|}{$\begin{array}{l}\text { Tension } \\
\text { Arousal }\end{array}$} \\
\hline & & & M & SD & M & SD & M & SD & M & SD & $\mathrm{M}$ & SD & M & SD & M & SD & M & SD \\
\hline 001 & Shallow Grave & 15 & 8.33 & 0.90 & 1.11 & 0.60 & 3.15 & 2.02 & 1.11 & 0.43 & 1.09 & 0.28 & 7.94 & 1.19 & 7.72 & 1.02 & 5.57 & 2.11 \\
\hline 003 & The Rainmaker & 18 & 7.35 & 1.04 & 1.30 & 0.79 & 3.85 & 2.17 & 1.09 & 0.35 & 1.15 & 0.56 & 7.49 & 1.12 & 6.89 & 0.94 & 4.81 & 1.99 \\
\hline 004 & $\begin{array}{l}\text { Man of Galilee } \\
\text { CD1 }\end{array}$ & 16 & 7.78 & 1.41 & 1.17 & 0.68 & 2.93 & 1.74 & 1.28 & 0.81 & 1.41 & 0.96 & 7.21 & 1.56 & 7.38 & 1.07 & 5.96 & 1.94 \\
\hline 010 & $\begin{array}{l}\text { The } \\
\text { Untouchables }\end{array}$ & 15 & 7.89 & 1.15 & 1.47 & 1.06 & 4.53 & 2.34 & 1.04 & 0.20 & 1.11 & 0.37 & 7.78 & 0.96 & 7.09 & 1.23 & 5.72 & 1.99 \\
\hline 011 & Shine & 16 & 6.78 & 2.02 & 1.63 & 1.40 & 3.96 & 2.43 & 1.48 & 1.03 & 1.22 & 0.51 & 6.72 & 1.19 & 6.98 & 1.61 & 5.49 & 1.91 \\
\hline 016 & Big Fish & 22 & 5.52 & 2.16 & 4.13 & 2.56 & 6.46 & 2.17 & 1.20 & 0.69 & 1.17 & 0.68 & 6.98 & 1.13 & 3.77 & 1.60 & 2.70 & 1.47 \\
\hline 018 & Shine & 19 & 6.68 & 1.63 & 2.13 & 1.68 & 4.47 & 2.30 & 1.87 & 1.58 & 1.81 & 1.33 & 6.89 & 1.42 & 6.80 & 1.34 & 5.57 & 1.93 \\
\hline 020 & Vertigo OST & 15 & 6.09 & 2.14 & 2.72 & 1.97 & 4.22 & 2.25 & 1.59 & 1.02 & 1.48 & 0.98 & 6.40 & 1.56 & 6.49 & 1.23 & 5.57 & 1.57 \\
\hline 021 & Gladiator & 13 & 5.83 & 2.03 & 2.63 & 1.97 & 4.67 & 2.33 & 1.46 & 1.15 & 1.30 & 0.84 & 6.47 & 1.67 & 5.15 & 1.56 & 4.36 & 2.02 \\
\hline 022 & $\begin{array}{c}\text { Shakespeare in } \\
\text { Love }\end{array}$ & 18 & 5.83 & 2.88 & 3.19 & 2.06 & 3.09 & 1.95 & 2.06 & 1.66 & 2.06 & 1.76 & 6.41 & 1.53 & 6.74 & 1.48 & 5.52 & 1.63 \\
\hline 029 & Vertigo OST & 15 & 3.11 & 1.93 & 5.79 & 2.18 & 4.74 & 2.51 & 2.23 & 1.72 & 2.02 & 1.62 & 5.41 & 1.87 & 4.28 & 1.97 & 3.93 & 2.05 \\
\hline 030 & $\begin{array}{l}\text { The English } \\
\text { Patient }\end{array}$ & 31 & 4.43 & 2.05 & 5.78 & 2.21 & 6.63 & 1.90 & 1.91 & 1.52 & 1.24 & 0.74 & 7.26 & 1.42 & 3.02 & 1.55 & 2.28 & 1.53 \\
\hline 032 & $\begin{array}{l}\text { The English } \\
\text { Patient }\end{array}$ & 25 & 1.67 & 1.19 & 7.89 & 1.27 & 4.89 & 2.77 & 2.00 & 1.89 & 1.54 & 1.17 & 5.26 & 2.06 & 3.15 & 1.63 & 2.96 & 1.72 \\
\hline 036 & $\begin{array}{l}\text { Running } \\
\text { Scared }\end{array}$ & 21 & 1.34 & 0.81 & 5.85 & 1.94 & 1.98 & 1.86 & 5.40 & 1.92 & 3.74 & 2.09 & 3.57 & 1.83 & 4.98 & 2.27 & 5.65 & 2.23 \\
\hline 037 & $\begin{array}{l}\text { Road To } \\
\text { Perdition }\end{array}$ & 25 & 1.51 & 1.04 & 6.34 & 2.20 & 2.40 & 1.86 & 3.93 & 2.25 & 2.87 & 2.26 & 3.93 & 1.70 & 3.91 & 2.18 & 4.50 & 2.03 \\
\hline
\end{tabular}




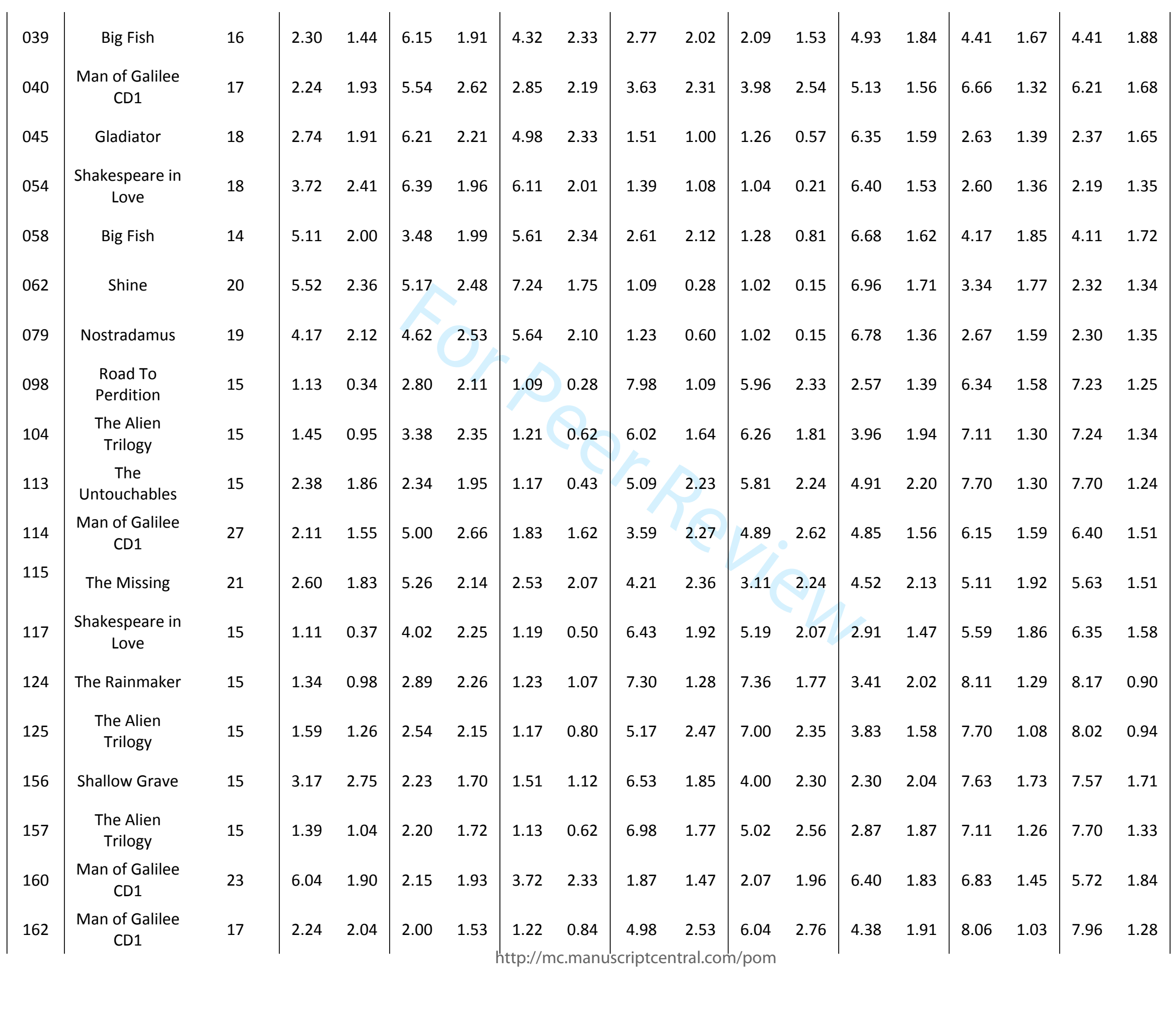




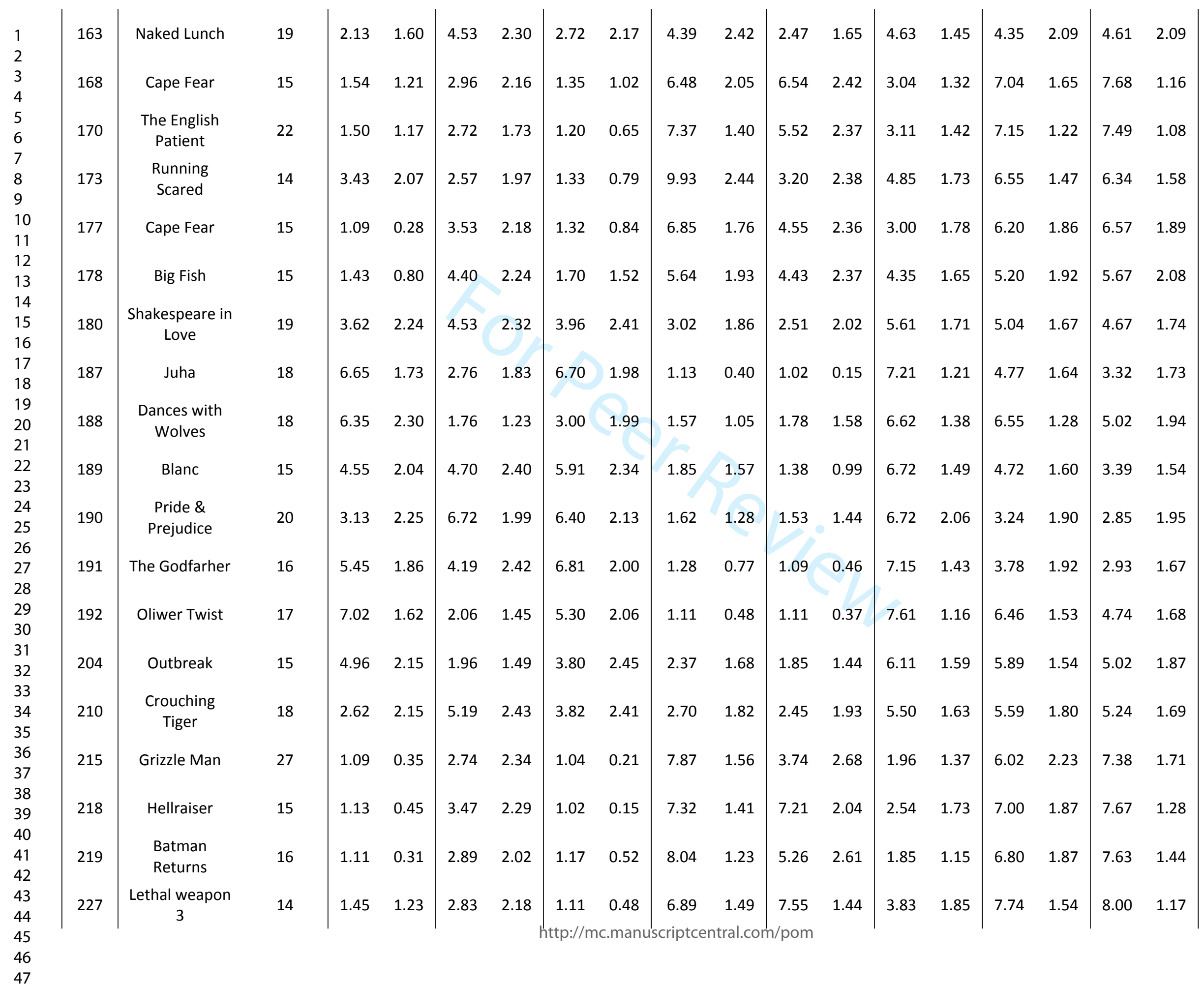




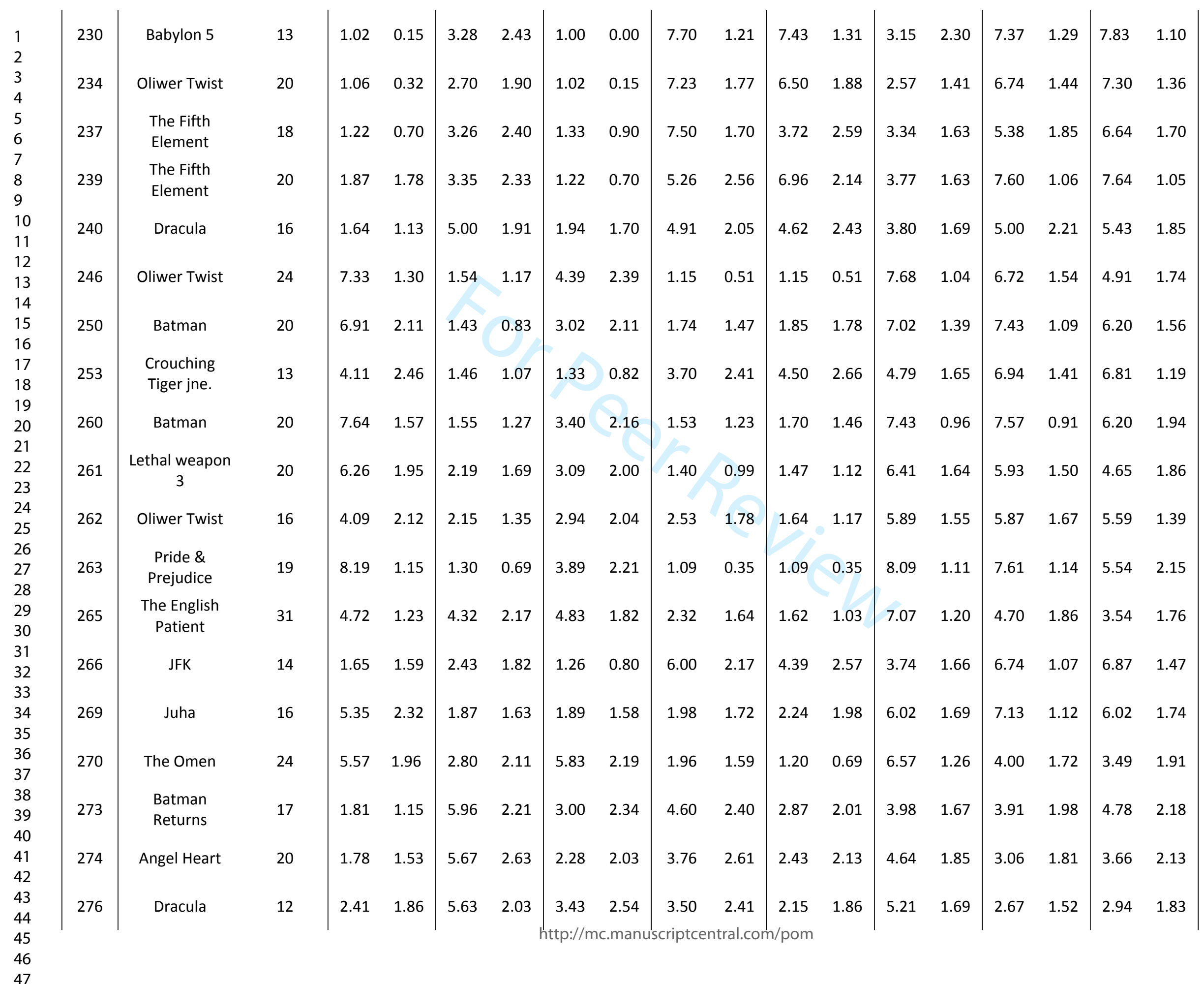




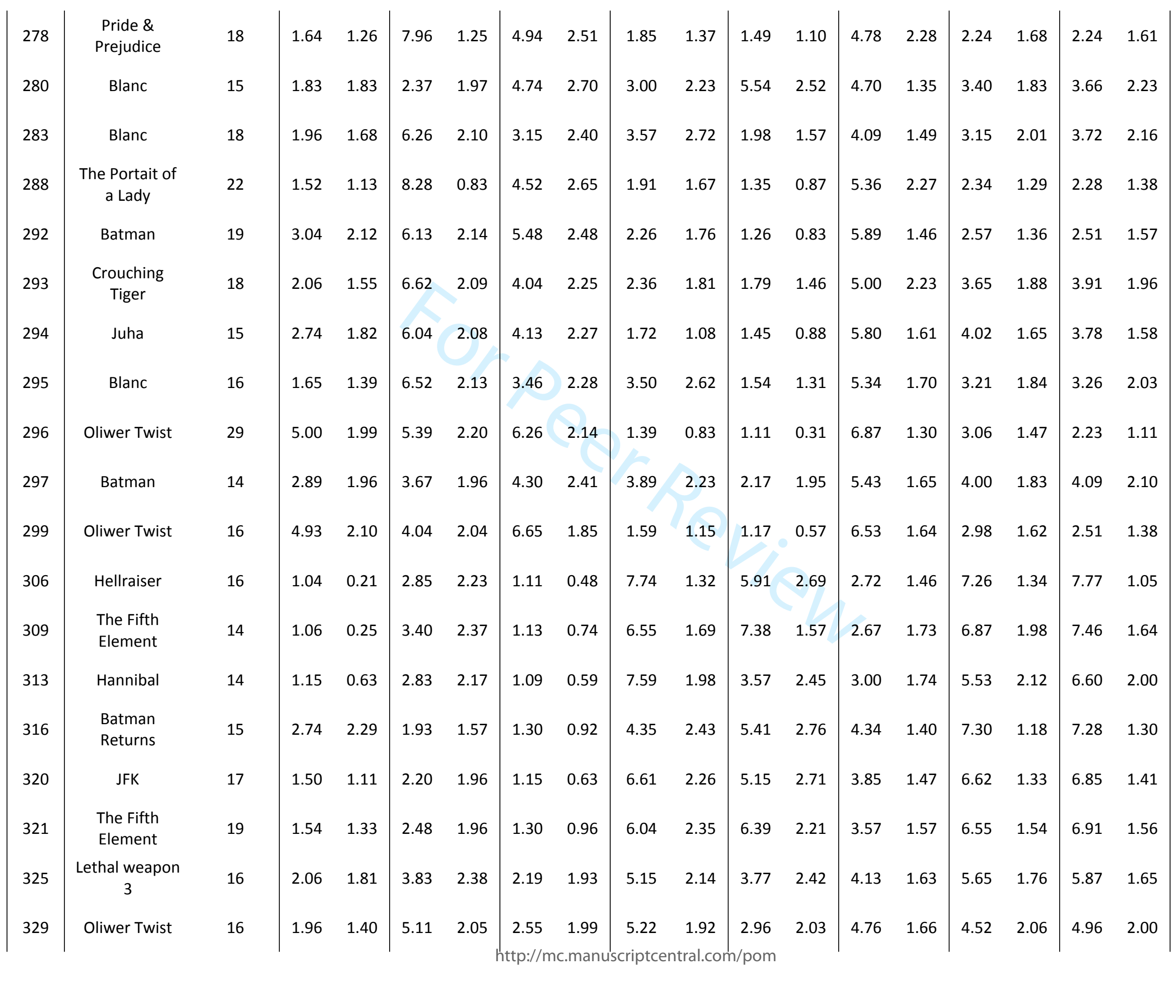




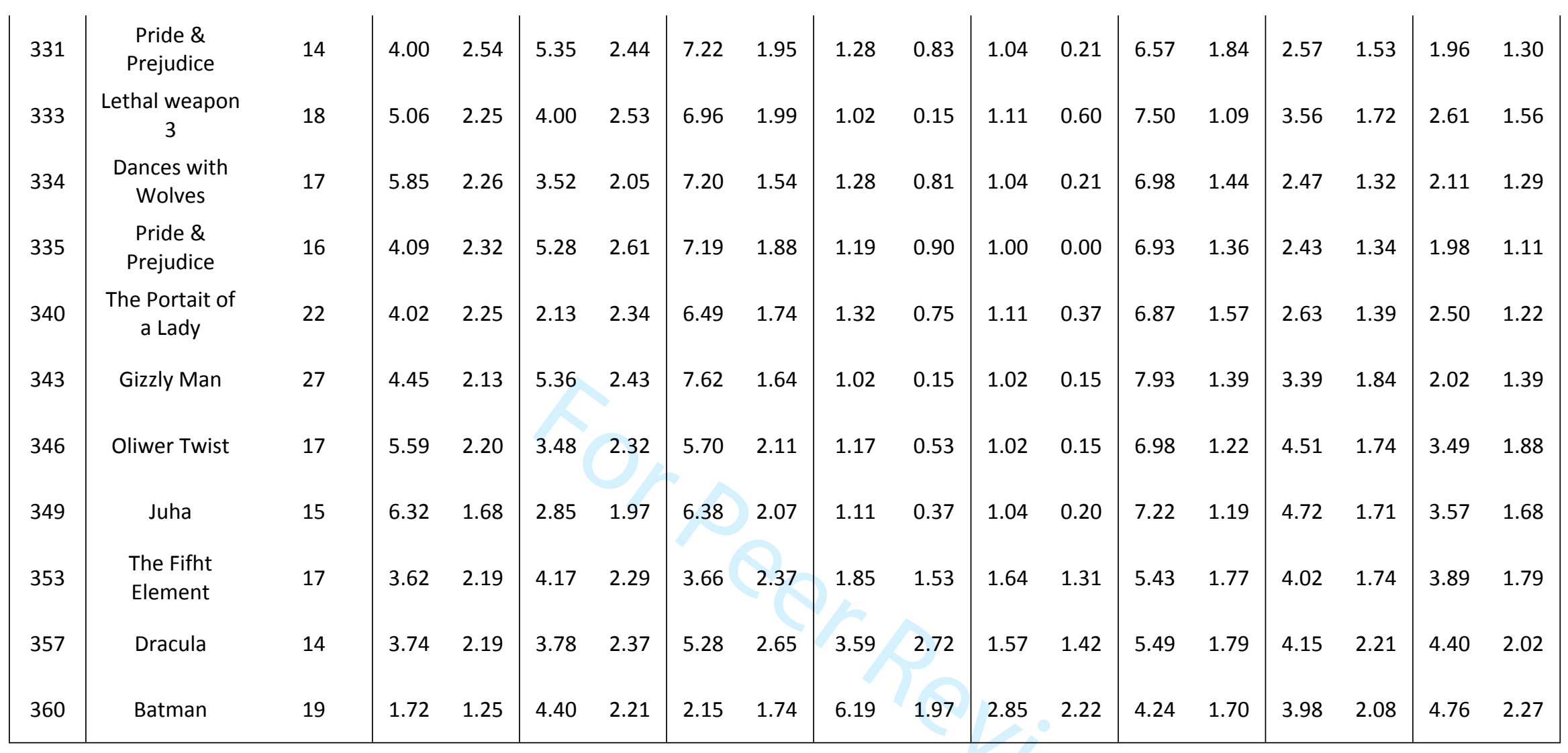


Appendix (Table 3). Mean (M) and Standard Deviation (SD) in the different affective scales for each excerpt for Spanish men $(n=37)$

\begin{tabular}{|c|c|c|c|c|c|c|c|c|c|c|c|c|c|c|c|c|c|c|}
\hline \multirow[b]{2}{*}{ № } & \multirow[b]{2}{*}{$\begin{array}{c}\text { Soundtrack } \\
\text { title }\end{array}$} & \multirow[b]{2}{*}{ Duration } & \multicolumn{2}{|c|}{ Happiness } & \multicolumn{2}{|c|}{ Sadness } & \multicolumn{2}{|c|}{ Tenderness } & \multicolumn{2}{|c|}{ Fear } & \multicolumn{2}{|c|}{ Anger } & \multicolumn{2}{|c|}{ Valence } & \multicolumn{2}{|c|}{$\begin{array}{l}\text { Energy } \\
\text { Arousal }\end{array}$} & \multicolumn{2}{|c|}{$\begin{array}{l}\text { Tension } \\
\text { Arousal }\end{array}$} \\
\hline & & & $\mathrm{M}$ & SD & $\mathrm{M}$ & SD & $\mathrm{M}$ & SD & $\mathrm{M}$ & SD & $\mathrm{M}$ & SD & M & SD & $\mathrm{M}$ & SD & M & SD \\
\hline 001 & Shallow Grave & 15 & 8.00 & 1.25 & 1.11 & 0.32 & 4.21 & 2.12 & 1.11 & 0.32 & 1.11 & 0.32 & 7.59 & 0.94 & 7.35 & 0.86 & 4.18 & 1.70 \\
\hline 003 & The Rainmaker & 18 & 7.21 & 0.98 & 1.53 & 0.90 & 5.05 & 1.87 & 1.26 & 0.45 & 1.16 & 0.37 & 7.06 & 0.90 & 5.82 & 1.59 & 3.59 & 1.42 \\
\hline 004 & $\begin{array}{c}\text { Man of Galilee } \\
\text { CD1 }\end{array}$ & 16 & 7.26 & 1.28 & 1.21 & 0.42 & 3.00 & 1.67 & 1.11 & 0.32 & 1.47 & 1.22 & 7.29 & 0.99 & 7.12 & 1.36 & 5.35 & 1.97 \\
\hline 010 & $\begin{array}{l}\text { The } \\
\text { Untouchables }\end{array}$ & 15 & 7.88 & 0.86 & 1.47 & 0.62 & 3.88 & 2.23 & 1.53 & 0.87 & 1.35 & 0.49 & 7.11 & 1.66 & 6.74 & 1.97 & 5.32 & 2.06 \\
\hline 011 & Shine & 16 & 6.58 & 1.74 & 1.42 & 0.77 & 4.47 & 2.32 & 1.58 & 1.12 & 1.32 & 1.00 & 6.76 & 1.25 & 6.59 & 1.00 & 5.12 & 1.45 \\
\hline 016 & Big Fish & 22 & 4.16 & 1.64 & 4.58 & 2.50 & 6.37 & 2.00 & 1.26 & 0.56 & 1.05 & 0.23 & 6.65 & 1.17 & 3.82 & 1.19 & 2.41 & 0.94 \\
\hline 018 & Shine & 19 & 6.35 & 1.97 & 2.47 & 1.74 & 5.18 & 2.30 & 2.12 & 1.54 & 1.71 & 1.26 & 6.26 & 1.69 & 6.53 & 1.47 & 6.11 & 1.85 \\
\hline 020 & Vertigo OST & 15 & 6.00 & 1.73 & 2.58 & 1.61 & 3.79 & 2.12 & 1.84 & 1.30 & 1.79 & 1.13 & 6.35 & 1.22 & 6.18 & 1.33 & 4.88 & 1.73 \\
\hline 021 & Gladiator & 13 & 5.16 & 2.22 & 2.42 & 1.46 & 4.26 & 2.05 & 1.58 & 0.77 & 1.42 & 1.02 & 6.71 & 1.16 & 5.29 & 1.49 & 3.71 & 1.72 \\
\hline 022 & $\begin{array}{c}\text { Shakespeare in } \\
\text { Love }\end{array}$ & 18 & 5.24 & 2.11 & 4.00 & 2.62 & 3.29 & 2.11 & 2.71 & 1.83 & 2.53 & 1.74 & 6.05 & 1.54 & 6.26 & 1.85 & 5.74 & 1.69 \\
\hline 029 & Vertigo OST & 15 & 3.76 & 2.08 & 5.65 & 2.21 & 5.24 & 2.49 & 2.76 & 1.75 & 2.00 & 1.73 & 6.00 & 1.76 & 4.05 & 1.72 & 4.37 & 1.83 \\
\hline 030 & $\begin{array}{c}\text { The English } \\
\text { Patient }\end{array}$ & 31 & 4.32 & 1.60 & 5.37 & 2.65 & 6.95 & 1.31 & 2.47 & 1.54 & 1.42 & 0.96 & 7.12 & 1.22 & 2.88 & 1.32 & 1.94 & 0.75 \\
\hline 032 & $\begin{array}{c}\text { The English } \\
\text { Patient }\end{array}$ & 25 & 1.89 & 1.56 & 6.95 & 1.51 & 4.95 & 2.80 & 1.95 & 1.27 & 1.37 & 0.83 & 4.76 & 1.56 & 2.88 & 1.36 & 2.82 & 1.07 \\
\hline 036 & $\begin{array}{l}\text { Running } \\
\text { Scared }\end{array}$ & 21 & 2.06 & 1.20 & 5.94 & 1.85 & 3.24 & 2.46 & 4.35 & 2.06 & 3.65 & 2.21 & 3.95 & 1.99 & 4.63 & 1.86 & 4.95 & 2.09 \\
\hline 037 & $\begin{array}{l}\text { Road To } \\
\text { Perdition }\end{array}$ & 25 & 1.88 & 1.27 & 6.71 & 1.69 & 3.82 & 2.48 & 4.12 & 2.03 & 3.71 & 2.37 & 4.63 & 2.14 & 3.53 & 1.43 & 4.00 & 2.08 \\
\hline
\end{tabular}




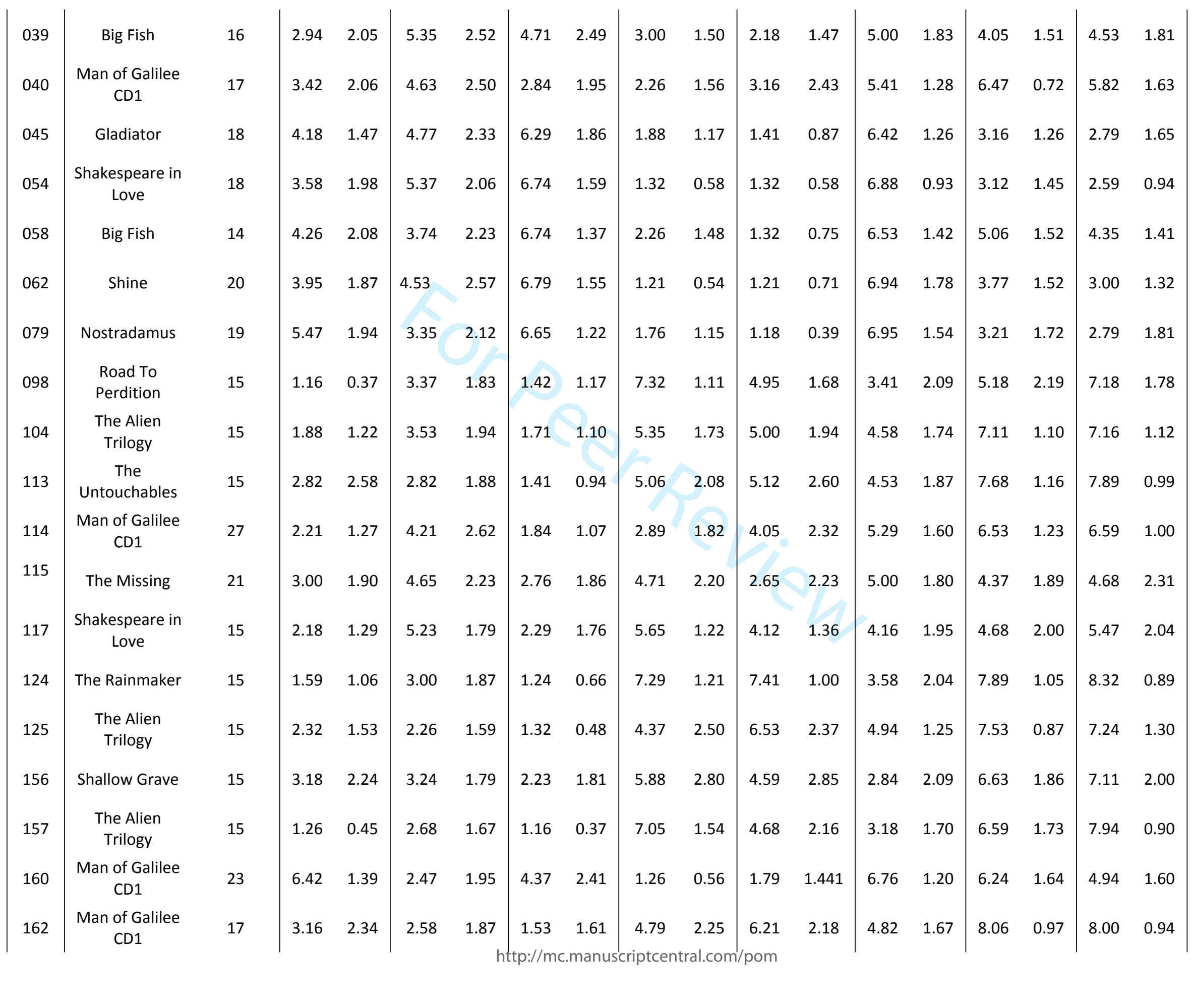




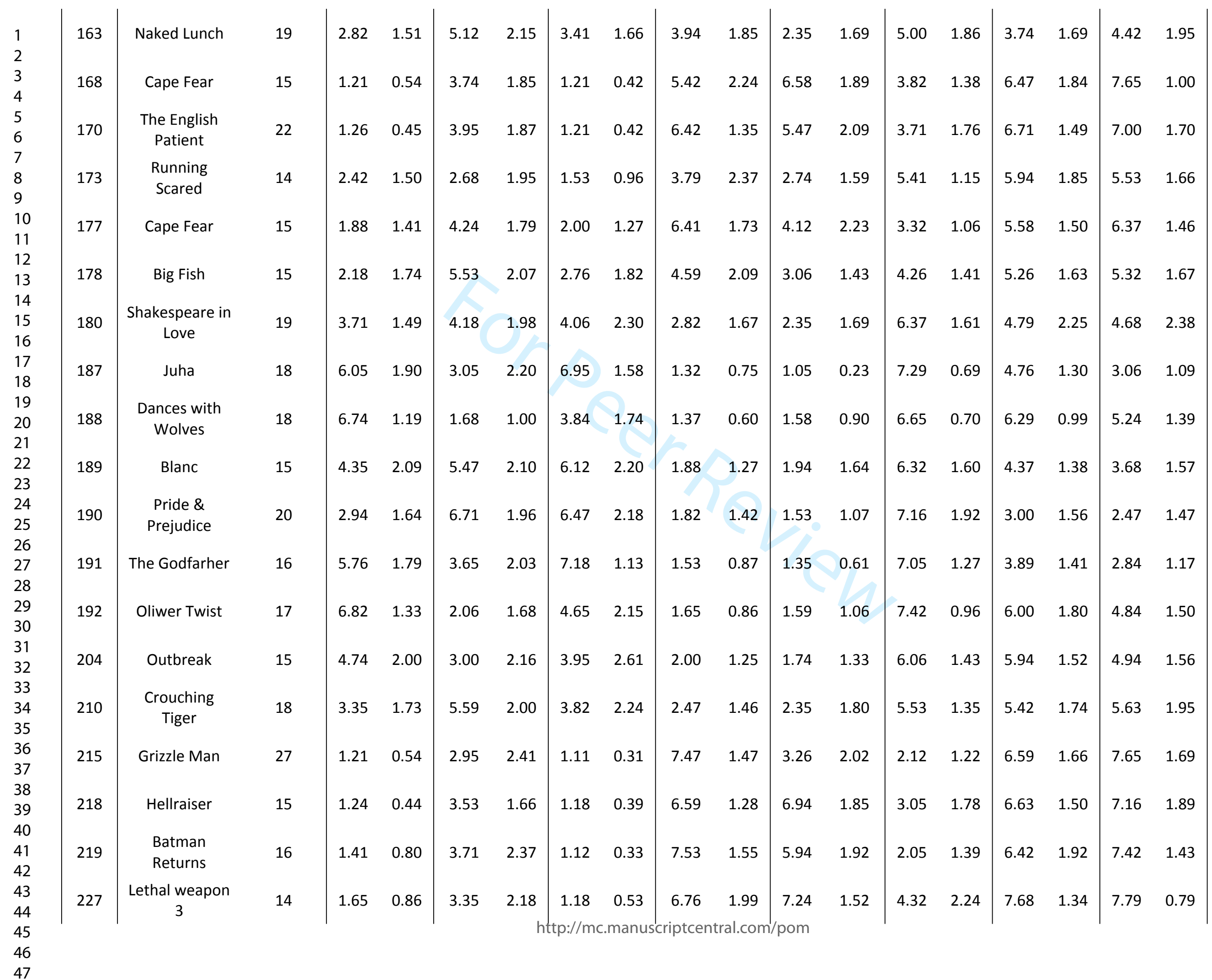




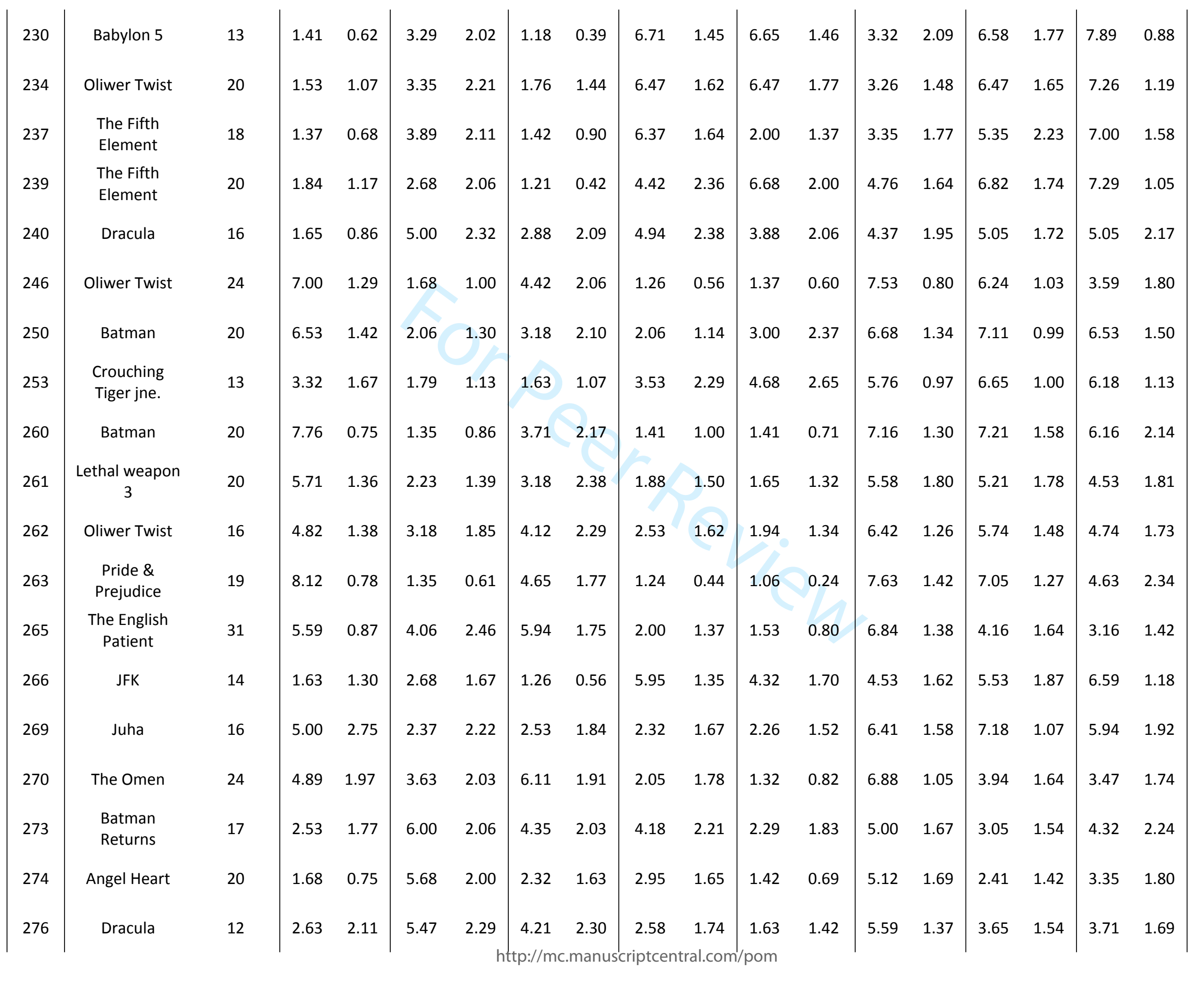




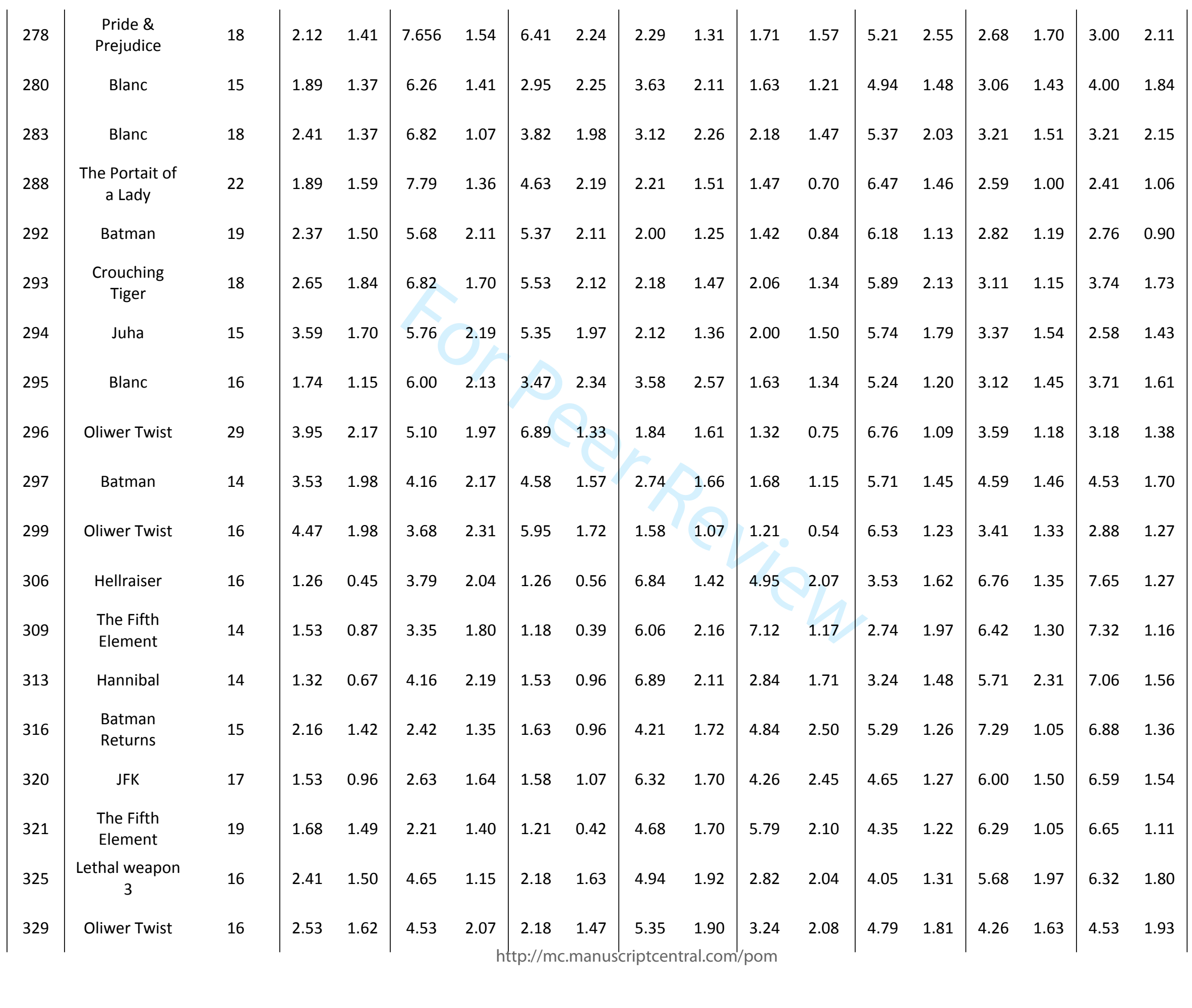




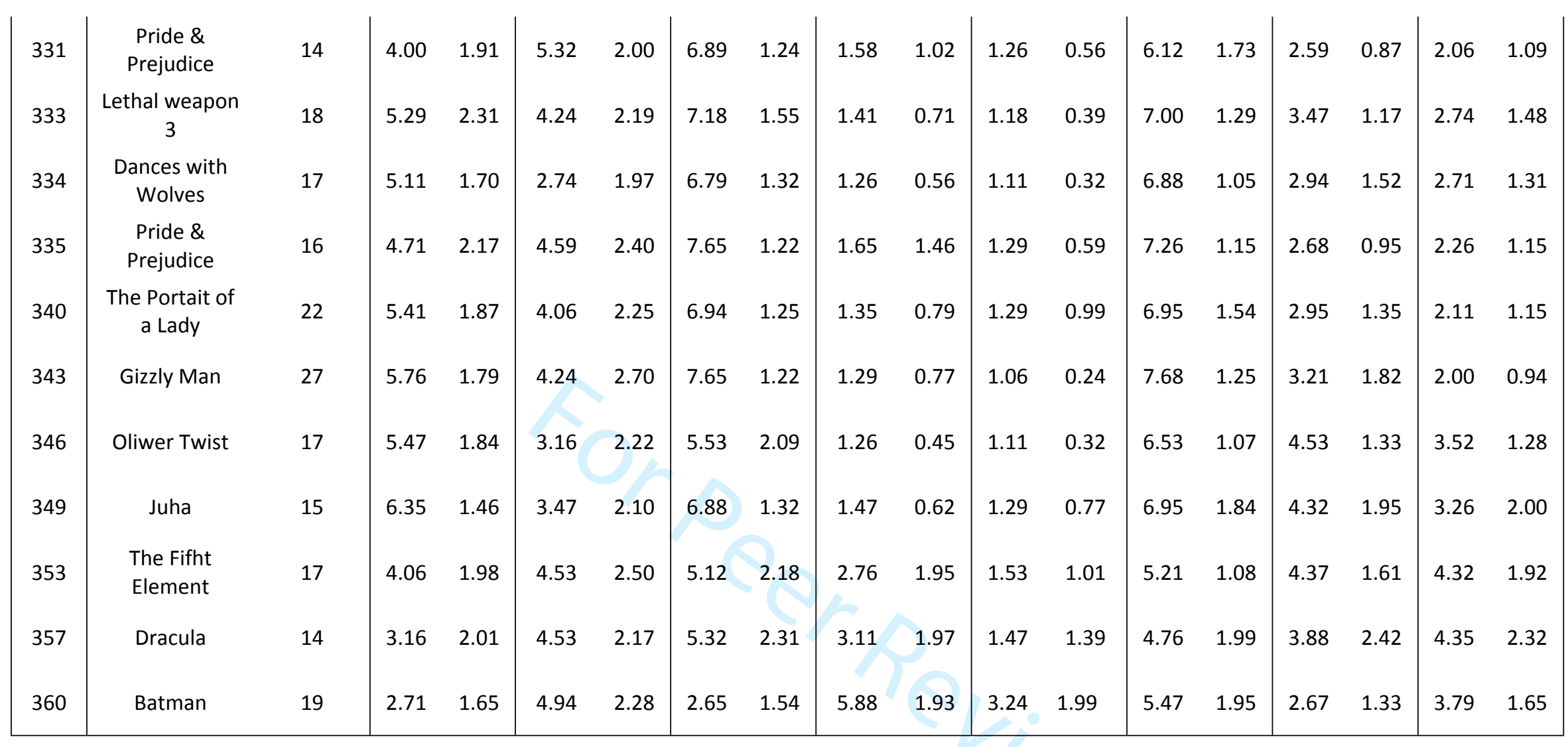

